\title{
DENSITY ESTIMATION OF LÉVY MEASURES FOR DISCRETELY OBSERVED DIFFUSION PROCESSES WITH JUMPS
}

\author{
Yasutaka Shimizu*
}

\begin{abstract}
We study a nonparametric estimation of Lévy measures for multidimensional jump-diffusion models from some discrete observations. We suppose that the jump term is driven by a Lévy process with finite Lévy measure, that is, a compound Poisson process. We construct a kernel-estimator of the Lévy density under a sampling scheme where the terminal time tends to infinity and at the same time the distance between the observations tends to zero fast enough, and show the $L^{2}$ consistency and the optimal rate in the MSE sense. First, we consider the case where the observations are given continuously and then compare it to the discretely observed case.
\end{abstract}

Key words and phrases: Consistency, discrete observations, jump-diffusion, kernel density estimation, Lévy density, MSE, optimal rate.

\section{Introduction}

We consider the following $k$-dimensional stochastic differential equation on a filtered probability space $\left(\Omega, \mathscr{F},\left(\mathscr{F}_{t}\right)_{t \geq 0}, P\right)$ :

$$
\left\{\begin{array}{l}
d X_{t}=a\left(X_{t-}\right) d t+b\left(X_{t-}\right) d W_{t}+\int_{E} c\left(X_{t-}, z\right)(p-q)(d t, d z), \\
X_{0}=x_{0}
\end{array}\right.
$$

where $E=\mathbb{R}^{d} \backslash\{0\},\left(W_{t}\right)_{t \geq 0}$ is a $k^{\prime}$-dimensional standard $\mathscr{F}_{t}$-Brownian motion, $a(x)$ is an $\mathbb{R}^{k}$-valued Borel function defined on $\mathbb{R}^{k}, b(x)$ is an $\mathbb{R}^{k} \otimes \mathbb{R}^{k^{\prime}}$-valued Borel function defined on $\mathbb{R}^{k}, c(x, z)$ is an $\mathbb{R}^{k}$-valued Borel function defined on $\mathbb{R}^{k} \times E, p(d t, d z)$ is a homogeneous Poisson random measure on $\mathbb{R}_{+} \times E$, and $q(d t, d z)$ is its intensity measure, that is, $E[p(d t, d z)]=q(d t, d z)$. We suppose that $q$ has the expression $q(d t, d z)=f(z) d z d t$, that is, $f$ is a Lévy density.

The solution process $X$ is called a diffusion process with jumps, or simply a jump-diffusion. Such models are used in many fields as flexible models to describe various phenomena. In particular, they are frequently used in financial modeling; it would be nice for the readers to refer to Prakasa Rao (1999) or Cont and Tankov (2004) and their references for details about applications. Therefore, inference of jump-diffusion models, especially from discrete observations, is of major importance in view of applications.

\footnotetext{
Received July 28, 2004. Revised October 24, 2004. Accepted October 31, 2005.

*Division of Mathematical Science, Graduate School of Engineering Science, Osaka University, Toyonaka, Osaka 560-8531, Japan.
} 
In this paper, we consider an estimation of the Lévy density $f(z)$ which is a crucial characteristic to determine properties of jumps under a nonparametric setting. We suppose that $\int_{\mathbb{R}^{d}} f(z) d z<\infty$ below, that is, jumps are controlled by a compound Poisson process. We provide a kernel type estimator of the Lévy density from some discrete observations.

When we deal with the discrete observations, we must determine the observation designs to discuss some asymptotic properties of estimators. Our design in this paper is that the terminal time of observation tends to infinity and the length of observation steps tends to zero.

Several authors have been studied the inference of diffusion processes under this sampling scheme in both nonparametric and parametric ways. For instance, Nguyen and Pham (1981) studied a nonparametric method under this scheme. In a parametric method, relatively general result for ergodic diffusions can be seen in Kessler (1997). See Prakasa Rao (2000) for an extensive review of inferences of usual diffusion type processes. However, we encounter a difficulty in the presence of jumps: it is impossible to identify the jumps exactly from only discrete observations. In other words, we can not discriminate a large increment caused by Brownian shocks from the one by a true discontinuity of the path.

In order to overcome this difficulty, Shimizu and Yoshida (2002) proposed a discrimination method. Their main idea was as follows. If an increment of neighboring data is large to some extent, then one judges that a single jump occurred in the interval, and if an increment is small then one judges that no jump occurred there. The probability with which more than two jumps occur is asymptotically ignored. The essence of their method is that one can regard a large increment as a jump size of the underlying processes approximately. We use such an approximated jump size to estimate the distribution of true ones.

Now, our procedure is nonparametric, in particular, kernel density method. If we can obtain continuous data of $X$ then it would be easy to construct consistent estimators of Lévy measures by the analogy of the case where samples are independent and identically distributed since we know all of amplitudes of jumps exactly. For example, we consider a one dimensional model:

$$
d X_{t}=a\left(X_{t-}\right) d t+b\left(X_{t-}\right) d W_{t}+d Z_{t},
$$

where $a, b$ are unknown functions, $W$ is a Wiener process, and $Z$ is a compound Poisson process with Poisson intensity 1 and unknown distribution of jumps $F(z) d z$. If we can observe the whole path on $[0, T]$, so we can observe the first $n$ jumps $\left(\Delta Z_{i}\right)_{1 \leq i \leq n}$ on $[0, T]$, then we can estimate the density $F(z)$ by

$$
F_{n}(z)=\frac{1}{n \delta_{n}} \sum_{i=1}^{n} K\left(\frac{z-\Delta Z_{i}}{\delta_{n}}\right)
$$

with a suitable kernel $K$ and a sequence $\delta_{n}$, which converges to the true $F$ in the MSE sense at the rate $n \delta_{n}$; see Masry (1983) for details. A more general type of inference from randomly sampled data is discussed in Prakasa Rao (1988, 1990). 
If we can observe discrete data only, it is natural to substitute the increments of neighboring data for $\Delta Z_{i}$ 's in above $F_{n}(z)$ according to the context of Shimizu and Yoshida (2002). Though we consider a more general type of stochastic differential equation, the same type of estimator can be available.

The plan of the paper is following. In Section 2, we prepare assumptions with some notations. In Section 3, we first consider an estimator from continuous observations and state an asymptotic property, and then we proceed to the discussion on an estimator from discrete observations. One can find that it will be a natural discretization of the continuous case. In Section 4, we give simulation results in some simple cases and point out some problems. In Section 5, we present a conclusion. The proofs of theorems are described in Section 6, and Appendix contains some useful technical results with their proofs.

\section{Notations and assumptions}

We suppose that the process $X$ is observed at each time point $t_{i}^{n}=i h_{n}$ $(i=1,2, \ldots, n)$ in the time interval $\left[0, T_{n}\right]$, where $T_{n}=n h_{n}$ with asymptotics $h_{n} \rightarrow 0, T_{n} \rightarrow \infty$ as $n \rightarrow \infty$. Our goal is to estimate the Lévy density $f(z)$ from such discrete observations. In the sequel, we use the following notations. Let $\mathscr{F}_{i-1}^{n}:=\mathscr{F}_{t-1}^{n}, \Delta X_{i}^{n}:=X_{t_{i}^{n}}-X_{t_{i-1}^{n}}$ and $\Delta X_{t}:=X_{t}-X_{t-}$. For a matrix $A,|A|^{2}$ is the sum of squares of the components of $A$. For a real sequence $u_{n}$, $R: \mathbb{R}^{d} \times \mathbb{R} \times \mathbb{R}^{k} \rightarrow \mathbb{R}$ denotes a function for which there exists a positive constant $C$ such that $R\left(z, u_{n}, x\right) \leq u_{n} C(1+|x|)^{C}$ for all $z \in \mathbb{R}^{d}, x \in \mathbb{R}^{k}$ and $n \in N$. $C_{r}^{m}(\ell)$ $(r=m+l, 0<l \leq 1, m \in N \cup\{0\})$ denotes the space of real valued bounded functions $f$, defined on $\mathbb{R}^{d}$, which are $m$ times differentiable and such that

$$
\left|\frac{\partial f^{(m)}}{\partial z_{1}^{j_{1}} \cdots \partial z_{d}^{j_{d}}}(z)-\frac{\partial f^{(m)}}{\partial z_{1}^{j_{1}} \cdots \partial z_{d}^{j_{d}}}\left(z^{\prime}\right)\right| \leq \ell\left|z-z^{\prime}\right|^{l}
$$

for every $z, z^{\prime} \in \mathbb{R}^{d} ; j_{1}+\cdots+j_{d}=m$. Moreover, we often use the notation $C$ (resp. $C_{k}$ ) as a universal positive constant (resp. depending on the index $k$ ), therefore we sometimes use the same character for different constants from line to line without specially mentioning.

Throughout this paper, we make the following four assumptions.

A 1. $f(z) \in C_{r}^{m}(\ell)$ for some $r>0$ and $m \in N \cup\{0\}$. Moreover $0<$ $\int_{E} f(z) d z<\infty$.

Under this assumption, jumps of $X$ occur finite times in a finite interval. In the discussion below, it is convenient to identify the Poisson random measure $p$ with a counting measure of jumps: $p(d t, d z)=\sum_{s \geq 0} \mathbf{1}_{\left\{\Delta Z_{s} \neq 0\right\}} \mathbf{1}_{\left(s, \Delta Z_{s}\right)}(d t, d z)$ for a compound Poisson process $Z_{t}=\sum_{i=1}^{N_{t}} \epsilon_{i}$ independent of $W$, where $N$ is a Poisson process with intensity $\lambda,\left(\epsilon_{i}\right)_{i \in \boldsymbol{N}}$ is an i.i.d. sequence of $d$-dimensional random vectors with a density $F(z)$. Then we have

$$
f(z)=\lambda F(z)
$$


as a well-known fact. We often use this notation below.

A 2. There exists a positive constant $L$ such that $|a(x)-a(y)|+\mid b(x)-$ $b(y)|\leq L| x-y \mid$. Moreover, the coefficient $c(x, z)$ is known, and there exists a function $\zeta(z)$ with $\int_{E} \zeta^{2}(z) f(z) d z<\infty$ such that $|c(x, z)-c(y, z)| \leq \zeta(z)|x-y|$ and $|c(x, z)| \leq \zeta(z)(1+|x|)$.

Remark 1. We admit that the coefficients $a(x)$ and $b(x)$ are unknown. The assumption that $c(x, z)$ is known seems to be very restrictive. However if we did not know $c(x, z)$, then it should not be possible to identify $\Delta Z$, therefore we can not estimate the distribution of $\Delta Z$. Nevertheless the readers may also be interested in considering the case where $c$ is unknown. In this setting, one can estimate, for example, an integral such as $\int_{E} c^{p}\left(X_{t-}, z\right) f(z) d z$ or $E\left[\int_{E} c^{p}\left(X_{t-}, z\right) f(z) d z\right]$; see Bandi and Nguyen (2003), Shimizu (2003).

The assumption A 2 ensures the existence and the uniqueness of the solution to the stochastic differential equation (1.1). For the details, see Jacod and Shiryaev (1987).

A 3. $\inf _{x}|c(x, z)| \geq c_{0}|z|$ for a constant $c_{0}>0$, and $y=c(x, z)$ has an inverse $z=c^{-1}(x, y)$ with bounded derivative with respect to $y$ for each $x$.

A 4. $\sup _{t} E\left[\left|X_{t}\right|^{p}\right]<\infty$ for arbitrary $p \geq 0$.

The assumption A 4 may be difficult to check this condition for general jumpdiffusion models. However it would be often indispensable in some inference procedures and so is our case. The existence of the higher order moments in stochastic differential equations with jumps should be studied further.

\section{The density estimation and optimal rate}

\subsection{Continuously observed case}

Before considering the estimation from sampled data, let us consider kernel density estimation for continuously observed diffusion processes with jumps.

In the following discussion, we consider the space $H_{m, l}$ of kernels of order $(m, l)(m \in N \cup\{0\}, 0<l \leq 1)$, that is, the space of mapping $K: \mathbb{R}^{d} \rightarrow \mathbb{R}$ bounded, integrable, with bounded derivative such that $\int_{\mathbb{R}^{d}} K(u) d u=1$ and satisfying the following conditions:

$$
\int_{\mathbb{R}^{d}}\left|\left(u_{1}, \ldots, u_{d}\right)\right|^{l}\left|u_{1}\right|^{\alpha_{1}} \cdots\left|u_{d}\right|^{\alpha_{d}}\left|K\left(u_{1}, \ldots, u_{d}\right)\right| d u_{1} \cdots d u_{d}<\infty
$$

for $\alpha_{1}, \ldots, \alpha_{d} \in N \cup\{0\} ; 0 \leq \alpha_{1}+\cdots+\alpha_{d} \leq m$, and

$$
\int_{\mathbb{R}^{d}} u_{1}^{\tilde{\alpha}_{1}} \cdots u_{d}^{\tilde{\alpha}_{d}} K\left(u_{1}, \ldots, u_{d}\right) d u_{1} \cdots d u_{d}=0
$$

for $\tilde{\alpha}_{1}, \ldots, \tilde{\alpha}_{d} \in N \cup\{0\} ; 1 \leq \tilde{\alpha}_{1}+\cdots+\tilde{\alpha}_{d} \leq m$.

Suppose that we can observe whole the path of $X$ in $[0, T]$, and let us denote by $\tau_{i}$ the time point of $i$ th jump of $X$. In the interval $[0, T]$, we can observe the 
samples $\left(\Delta Z_{\tau_{i}}\right)_{1 \leq i \leq N_{T}}$, where $\Delta Z_{s}=c^{-1}\left(X_{s-}, \Delta X_{s}\right)$, and we can regard them as the randomly observed samples, whose number $N_{T}$ follows a Poisson distribution with an intensity $\lambda T$, from a stationary process with the marginal distribution $F$. Our interest is to estimate the function $f(z)=\lambda F(z)$ from the above samples. A possible estimator is given by the following intuitive discussion: As well as the i.i.d. case, a kernel estimator of $F$ may be given by

$$
\begin{aligned}
F_{T}(z) & :=\frac{1}{N_{T} \delta_{T}^{d}} \sum_{i=1}^{N_{T}} K\left(\frac{z-\Delta Z_{\tau_{i}}}{\delta_{T}}\right) \\
& =\frac{1}{N_{T} \delta_{T}^{d}} \int_{0}^{T} K\left(\frac{z-\Delta Z_{t}}{\delta_{T}}\right) d N_{t},
\end{aligned}
$$

where $K \in H_{m, l}$ and $\delta_{T}$ is a real sequence which satisfies some conditions. Since $N_{T} / T \rightarrow \lambda(T \rightarrow \infty)$ with probability one, and amplitude of each jump is independent of a Poisson process $N$,

$$
f_{T}(z):=\frac{N_{T}}{T} F_{T}(z)=\frac{1}{T \delta_{T}^{d}} \int_{0}^{T} K\left(\frac{z-\Delta Z_{t}}{\delta_{T}}\right) d N_{t}
$$

may be expected to become an $L^{2}$-consistent estimator of $f(z)$. Actually, Prakasa Rao $(1988,1990)$ studied the same problem as $d=1$. He studied some asymptotic properties of the same type of estimators made by delta-type kernels. As $d=1$, our kernel corresponds to his special case but we deal with a multidimensional case. The following theorem shows the consistency in an MSE sense and the optimal rate of convergence.

TheOREM 3.1. (Optimal rate in continuously observed case) Assume that there exists a real-valued sequence $\left\{\delta_{T}\right\}$ indexed by $T$ such that $\eta_{T}:=$ $\delta_{T} T^{1 /(2 r+d)} \rightarrow \eta$ for a positive constant $\eta$. Then, the following inequality is valid for the estimator $f_{T}(z)$ given in (3.3):

$$
\limsup _{T \rightarrow \infty} \sup _{z \in \mathbb{R}^{d}} T^{2 r /(2 r+d)} E\left|f_{T}(z)-f(z)\right|^{2} \leq C(\eta, f, K),
$$

where

$$
\begin{aligned}
C(\eta, f, K)= & \eta^{2 r}\left(\sum_{j_{1}+\cdots+j_{d}=m} \frac{\ell}{j_{1} ! \cdots j_{d} !} \int_{\mathbb{R}^{d}}|u|^{l}\left|u_{1}\right|^{j_{1}} \cdots\left|u_{d}\right|^{j_{d}}|K(u)| d u\right)^{2} \\
& +\eta^{-d} \sup _{z \in \mathbb{R}^{d}}|f(z)| \int_{\mathbb{R}^{d}} K^{2}(u) d u
\end{aligned}
$$

Remark 3.1. The convergence rate $T^{2 r /(2 r+d)}$ is natural as an analogy to the i.i.d. case; see Ibragimov and Has'minskii (1981), Chapter IV. If $d=1$ then this rate is consistent with the case of the delta-kernel $\delta_{T}^{-1} K\left(z \delta_{t}^{-1}\right)$ as in Prakasa Rao (1990), and this is a natural extension to the multidimensional case. 


\subsection{Discretely observed case}

As described in Section 1, we use the judgment by Shimizu and Yoshida (2002) to discriminate the continuity from the discontinuity of the path in each $h_{n}$-time interval. Their idea is as follows. For a critical value $h_{n}^{\rho}$ for a constant $\rho \in$ $[0,1 / 2)$, one can judge that there is a jump in an interval $\left[t_{i-1}^{n}, t_{i}^{n}\right]$ if $\left|\Delta X_{i}^{n}\right|>h_{n}^{\rho}$, and that there is no jump in the interval if $\left|\Delta X_{i}^{n}\right| \leq h_{n}^{\rho}$, moreover it is possible to ignore the event with more than two jumps in the interval if $h_{n}$ is sufficiently small.

The following lemma which justifies the above judgments is a corollary of the lemma 2.2 in Shimizu and Yoshida (2002), and the proof is almost the same as the original one, therefore we omit the details of the proof.

LEMMA 3.1. Let $J_{i}^{n}$ be the number of jumps in an interval $\left[t_{i}^{n}, t_{i-1}^{n}\right)$ and we set

$$
\left\{\left|\Delta X_{i}^{n}\right| \leq L h_{n}^{\rho}\right\}=\bigcup_{j=0}^{2} C_{i, j}^{n}, \quad\left\{\left|\Delta X_{i}^{n}\right|>L h_{n}^{\rho}\right\}=\bigcup_{j=0}^{2} D_{i, j}^{n}
$$

for constants $L>0$ and $\rho \in[0,1 / 2)$, where

$$
\begin{array}{ll}
C_{i, 0}^{n}=\left\{J_{i}^{n}=0,\left|\Delta X_{i}^{n}\right| \leq L h_{n}^{\rho}\right\}, & D_{i, 0}^{n}=\left\{J_{i}^{n}=0,\left|\Delta X_{i}^{n}\right|>L h_{n}^{\rho}\right\}, \\
C_{i, 1}^{n}=\left\{J_{i}^{n}=1,\left|\Delta X_{i}^{n}\right| \leq L h_{n}^{\rho}\right\}, & D_{i, 1}^{n}=\left\{J_{i}^{n}=1,\left|\Delta X_{i}^{n}\right|>L h_{n}^{\rho}\right\}, \\
C_{i, 2}^{n}=\left\{J_{i}^{n} \geq 2,\left|\Delta X_{i}^{n}\right| \leq L h_{n}^{\rho}\right\}, & D_{i, 2}^{n}=\left\{J_{i}^{n} \geq 2,\left|\Delta X_{i}^{n}\right|>L h_{n}^{\rho}\right\} .
\end{array}
$$

Then, for any $p \geq 1$,

$$
\begin{aligned}
& P\left\{C_{i, 0}^{n} \mid \mathscr{F}_{i-1}^{n}\right\}=e^{-\lambda h_{n}} \tilde{R}\left(z, h_{n}^{p}, X_{t_{i-1}^{n}}\right), \\
& P\left\{D_{i, 0}^{n} \mid \mathscr{F}_{i-1}^{n}\right\}=e^{-\lambda h_{n}} R\left(z, h_{n}^{p}, X_{t_{i-1}^{n}}\right), \\
& P\left\{C_{i, 1}^{n} \mid \mathscr{F}_{i-1}^{n}\right\}=R\left(z, h_{n}^{\rho+1}, X_{t_{i-1}^{n}}\right), \\
& P\left\{D_{i, 1}^{n} \mid \mathscr{F}_{i-1}^{n}\right\}=\lambda h_{n} e^{-\lambda h_{n}} \tilde{R}\left(z, h_{n}^{\rho}, X_{t_{i-1}^{n}}\right), \\
& P\left\{C_{i, 2}^{n} \mid \mathscr{F}_{i-1}^{n}\right\} \leq \lambda^{2} h_{n}^{2}, \quad P\left\{D_{i, 2}^{n} \mid \mathscr{F}_{i-1}^{n}\right\} \leq \lambda^{2} h_{n}^{2},
\end{aligned}
$$

where $\tilde{R}\left(z, u_{n}, x\right)=1-R\left(z, u_{n}, x\right)$.

The order of the probability of $C_{i, 1}^{n}$ in the above lemma is different from the one of order $h_{n}^{3}$ in Shimizu and Yoshida (2002). They assumed that a Lévy density $f(z)$ satisfies $|f(z)| \leq c|z|^{\gamma}(\gamma>3)$ around the origin for estimation under the asymptotics $n h_{n}^{2} \rightarrow 0$. However, we only assume the boundedness of $f$ around the origin. Therefore, it is possible in our setting that small jumps occur more frequently than their setting, and it would be more difficult to identify such a small jumps. That is why the order of the probability of $C_{i, 1}^{n}$ is smaller than the one in Shimizu and Yoshida (2002). On the other hand, we have to demand more rapid experimental design such that $n h_{n}^{1+\delta} \rightarrow 0$ for a constant $\delta \in(0,1 / 2]$ instead of relaxing the assumption on Lévy density.

Remark 3.2. One might think that the constant $L$ in the filters is redundant since it could be included in the sequence $h_{n}$ by regarding $h_{n}$ as $L^{-1 / \rho} h_{n}$. 
Nevertheless it is convenient to leave this constant $L$ since $h_{n}$ is the observation interval, which is given in practical data, and we construct the filters in accordance with them. Furthermore we encounter a problem that the filters do not work well when $n$ is fixed in dealing with the practical data. Selecting $L$ suitably, we can improve the performance of estimation; see Section 4.

According to the context in Shimizu and Yoshida (2002), the amplitude of jump of $X$ in the interval $\left[t_{i-1}^{n}, t_{i}^{n}\right)$ can be approximated by the increment $\Delta X_{i}^{n}$. Therefore, we can estimate unobservable jumps of $Z$ as $\Delta Z_{i}^{n}:=c^{-1}\left(X_{t_{i-1}^{n}}, \Delta X_{i}^{n}\right)$, and it would be natural to use the following $f_{n}(z)$ as an estimator of the Lévy density:

$$
f_{n}(z):=\frac{1}{T_{n} \delta_{n}^{d}} \sum_{i=1}^{n} K\left(\frac{z-\Delta Z_{i}^{n}}{\delta_{n}}\right) \mathbf{1}_{\left\{\left|\Delta X_{i}^{n}\right|>L h_{n}^{\rho}\right\}},
$$

where $K \in H_{m, l}$ and $\left(\delta_{n}\right)_{n \geq 1}$ is a real sequence such that $T_{n} \delta_{n}^{d} \rightarrow \infty$. This is a straight discretization of (3.3).

Our main theorem is the following.

Theorem 3.2. (Optimal rate in discretely observed case) Assume that there exists a constant $\nu \in\left(0,2^{-1}(2 r+d)(2 r+d+1)^{-1}\right)$ such that $T_{n} h_{n}^{\nu}=O(1)$ as $n \rightarrow \infty$, and that there exist a constant $\rho \in[0,1 / 2)$ and a real-valued sequence $\left\{\delta_{n}\right\}$ indexed by $n$ such that $\eta_{n}:=\delta_{n} T_{n}^{1 /(2 r+d)} \rightarrow \eta$ for a positive constant $\eta$, $\delta_{n} h_{n}^{\rho-1 / 2}=O(1)$ and $T_{n} h_{n}^{1 / 2} \delta_{n}^{r-1}=o(1)$ as $n \rightarrow \infty$. Then the following inequality is valid for the estimator $f_{n}(z)$ given in (3.5):

$$
\limsup _{n \rightarrow \infty} \sup _{z \in \mathbb{R}^{d}} T_{n}^{2 r /(2 r+d)} E\left|f_{n}(z)-f(z)\right|^{2} \leq D(\eta, f, K),
$$

where

$$
\begin{aligned}
D(\eta, f, K)= & \eta^{2 r}\left(\sum_{j_{1}+\cdots+j_{d}=m} \frac{\ell}{j_{1} ! \cdots j_{d} !} \int_{\mathbb{R}^{d}}|u|^{l}\left|u_{1}\right|^{j_{1}} \cdots\left|u_{d}\right|^{j_{d}}|K(u)| d u\right)^{2} \\
& +\eta^{-d} \sup _{z \in \mathbb{R}^{d}}|f(z)| \int_{\mathbb{R}^{d}} K^{2}(u) d u
\end{aligned}
$$

Remark 3.3. The above convergence rate $T_{n}^{2 r /(2 r+d)}$ is natural since this attains the optimal rate for continuous case. However, without being particular about the optimality, it is possible to improve the order of the experimental design, that is, it is also possible to estimate under the assumption, for example, $T_{n} h_{n}^{\nu} \rightarrow \infty$ but $T_{n} h_{n}^{\tilde{\nu}} \rightarrow 0$ for some $\tilde{\nu} \geq 1 / 2$; see the expression (6.3) below.

Remark 3.4. We sometimes can not check the condition A 4. However this condition is one of the sufficient conditions for the above theorem and the estimator $f_{n}(z)$ sometimes can work well without this condition if we construct the filters suitably. In Subsection 4.4 below, we describe how to construct the 
another filter under the case where the diffusion coefficient $b$ is known, and show the performance for that filters.

\section{Simulation study}

For the simulation study, we use the following one dimensional stochastic differential equation:

$$
d X_{t}=\mu X_{t} d t+b\left(X_{t}, \sigma\right) d W_{t}+d Z_{t}, \quad Z_{t}=\sum_{j=1}^{N_{t}} \epsilon_{j},
$$

where $N_{t}$ is a Poisson process with intensity $\lambda$ and $\epsilon_{j}$ 's are i.i.d. r.v's with a density $F(z)$ which satisfies A 1 .

In Subsections 4.1-4.3 below, we consider the case $b(x, \sigma)=\sigma$ in which $X$ is ergodic and there exists an invariant measure $\pi$ if $\mu<0$. This process is called a Lévy driven Ornstein-Uhlenbeck (O-U) process. It is known that $\sup _{t} E\left[\left|X_{t}\right|^{p}\right]<\infty$ if, for any $p>0, \int_{E}|z|^{p} f(z) d z<\infty$ and $\int_{\mathbb{R}^{d}}|x|^{p} \eta(d x)<\infty$ for $\eta$ which is the distribution of $X_{0}$; see Masuda (2004), therefore, A 4 are satisfied in this model. Of course, the assumptions A 2-A 3 are satisfied.

In Subsection 4.4 below, we set $b(x, \sigma)=\sigma x$ in which $X$ is non-ergodic. It is not so easy to check A 4 , and the condition would be probably unsatisfied. However, we show that our method is robust without this condition if we choose some suitable filters. In this example, we assume that the diffusion coefficient is known, and we propose another filter which is data adaptive.

In each simulation below, we computed the estimated value $f_{n}(z)$ as a pointwise sample mean based on 500 times experiments. We set $X_{0}=1.0, h_{n}=n^{-0.8}$ and $K(x)=\frac{1}{\sqrt{2 \pi}} e^{-x^{2} / 2}$ as a kernel throughout this section.

\subsection{Simple examples}

First, let us consider the case where $F(z)$ is the density of Gaussian distribution $\mathcal{N}(0, \nu)$ and $(\mu, \sigma, \lambda, \nu)=(-0.01,0.01,15.0,1.0)$. We choose $\rho=0.49$, $\delta_{n}=n^{-0.1}$ and $L=1.0$. The estimation results are reported in Figure 1. It will be comparatively easy to judge whether a jump occurred or not in this model; if a jump occurred, then it should be large compared with the Brownian shocks. We see from Figure 1 that the variance of jumps seems to be overestimated since the small jumps are cut by the filters.

Second, we give another example for the model (4.1) with $(\mu, \sigma, \lambda)=$ $(-0.03,0.01,1.0), \rho=0.49$, but $F(z)$ is a density of the two-sided Gamma distribution $\Gamma(\alpha, \beta)$ :

$$
F(z)=\frac{1}{2} \beta^{\alpha}|x|^{\alpha-1} \Gamma^{-1}(\alpha) \exp (-\beta|x|) .
$$

with $\alpha=4.0$ and $\beta=5.0$. We show the results in Figure 2 with the sample sizes $n=500,3000,10000$, and 15000, respectively. In this simulation, we set $L=0.2$ in the filters and this seems to discriminate the pure jumps from the diffusion shocks so well. 


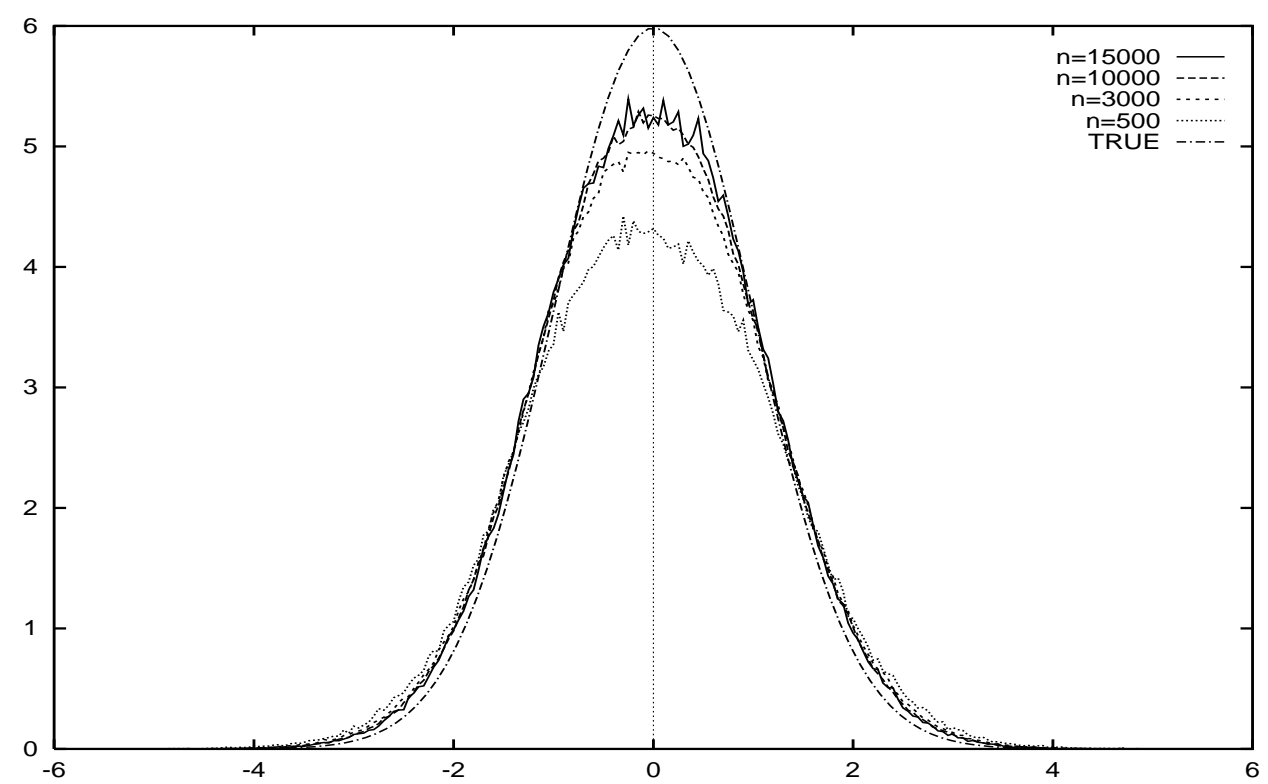

Figure 1. Estimation of the Lévy density in the model $f(z)=\lambda(\sqrt{2 \pi \nu})^{-1} \exp \left(-(2 \nu)^{-1} z^{2}\right)$ with $(\mu, \sigma, \lambda, \nu)=(-0.01,0.01,15.0,1.0), \rho=0.49, L=1.0$ for sample size $500,3000,10000$ and 15000 , respectively.

\subsection{Some troubles in estimation}

In the first two examples, we took $L=1.0$ in the former example and $L=0.2$ in the latter, and that $\rho=0.49$ in common. How should we choose the constant $L$ and $\rho$ ?

Thanks to the Lemma 3.1, it may be better to choose $\rho \in[0,1 / 2)$ as large as possible since the larger $\rho$ becomes, the more easily the filters can judge a single jump. Hence we consider the choice of $L$ with $\rho$ fixed as large as possible; in the sequel, we fix $\rho=0.49$.

On the constant $L$, we might have no way to choose $L$ by the asymptotic theory. However, if the constant $L$ is chosen unsuitably, then the performance of estimation can get worse. See Figure 3. They are the results in which we chose the true value of the diffusion parameter $\sigma(=0.5)$ larger than the one in the former example. The other setting is the same.

In this case, the estimated densities are awfully overestimated, particularly near the origin. It seems that the filters misunderstood the large increments by Brownian shocks with by jumps, so the Poisson intensity is overestimated. This is the trouble caused by the observation number $n$ stopped.

Our estimator $f_{n}(z)$ is theoretically $L^{2}$-consistent estimator and Theorem 3.2 ensures that our filters asymptotically work well for any selection of $L$. However, the sufficient sample number can depend on the structure of the true model, therefore we have to choose the suitable constant $L$ according to $n$ in each model. Of course, this dose not imply that some choices of $L$ possibly lead to incon- 


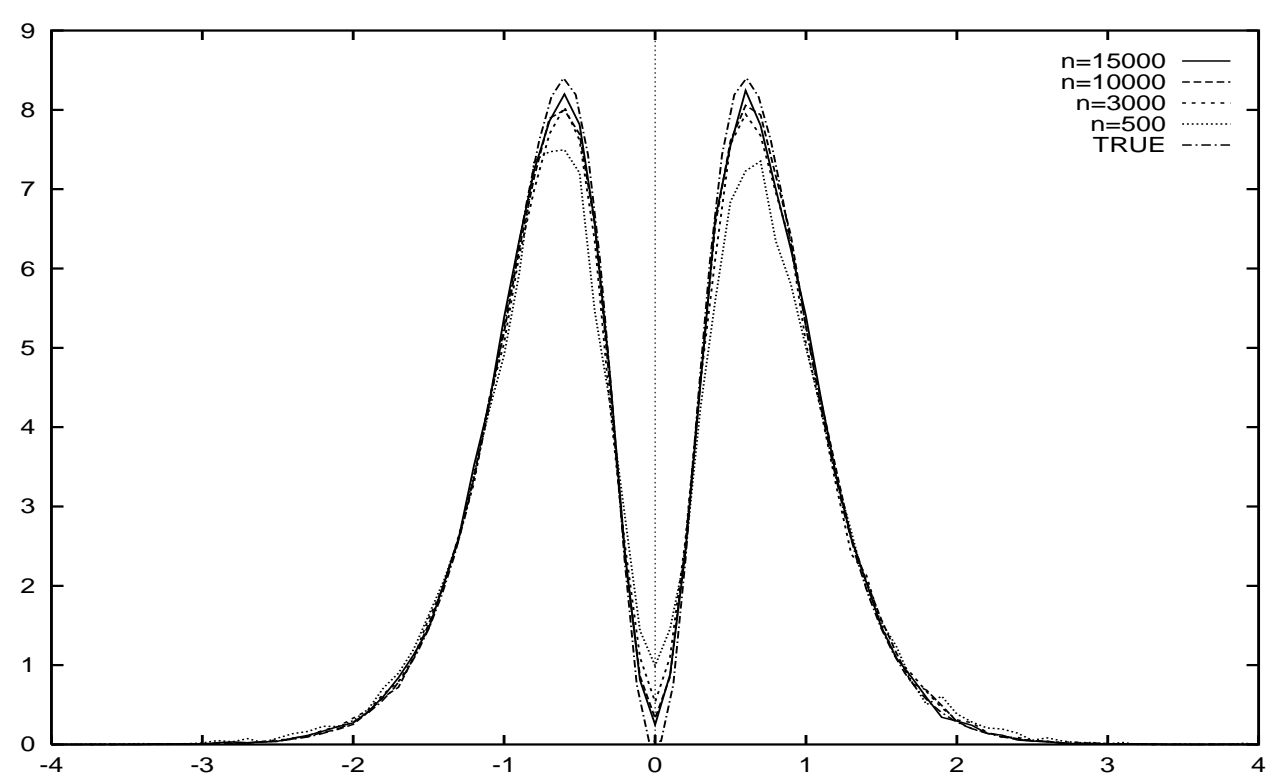

Figure 2. Estimation of the Lévy density in the model $F(z)=2^{-1} \beta^{\alpha}|x|^{\alpha-1} \Gamma^{-1}(\alpha) \exp (-\beta|x|)$ with $(\mu, \sigma, \lambda, \alpha, \beta)=(-0.03,0.01,15.0,4.0,5.0), \rho=0.49, L=0.2$ for sample size 500, 3000, 10000 and 15000 , respectively.

sistency of the estimator; this can be confirmed, for example, in Figure 6 as mentioned later.

\subsection{Selection problem of filters}

According to the discussion in Shimizu and Yoshida (2002), if the process $X$ is ergodic, the estimator

$$
\hat{\lambda}_{n}(L)=\frac{1}{n h_{n}} \sum_{j=1}^{n} \mathbf{1}_{\left\{\left|\Delta X_{i}^{n}\right|>L h_{n}^{\rho}\right\}}
$$

can be one of estimators of the intensity $\lambda$. Figure 4 shows the graphs of the $L$-pointwise sample mean of $\hat{\lambda}_{n}(L)$ based on 1000 simulations for $n=500,3000$ and 10000; it corresponds to the approximation of the curve of $E\left[\hat{\lambda}_{n}(L)\right]$. It is natural that $\hat{\lambda}_{n}(L)$ decrease rapidly in small $L$, and slowly in large $L$ since the filters for small $L$ capture the increments by diffusions, whose order of numbers is $O(n)$, as well as by the true jumps, and the filters for large $L$ hardly capture the increments by diffusions but some large jumps, whose order of numbers is $O\left(n h_{n}\right)$. Therefore, intuitively speaking, it might be better to choose the smallest possible $L$ at which the curve becomes nearly flat. It implies that the filters excluded influences by diffusions as much as possible, but does not exclude too much the true jumps. To see this, we reported in Figure 5 the graphs of the numerical derivatives in $L$ of the estimated intensity curve. In order to avoid blurring of the pointwise calculated numerical derivatives, we plotted the five-points moving average curves. 


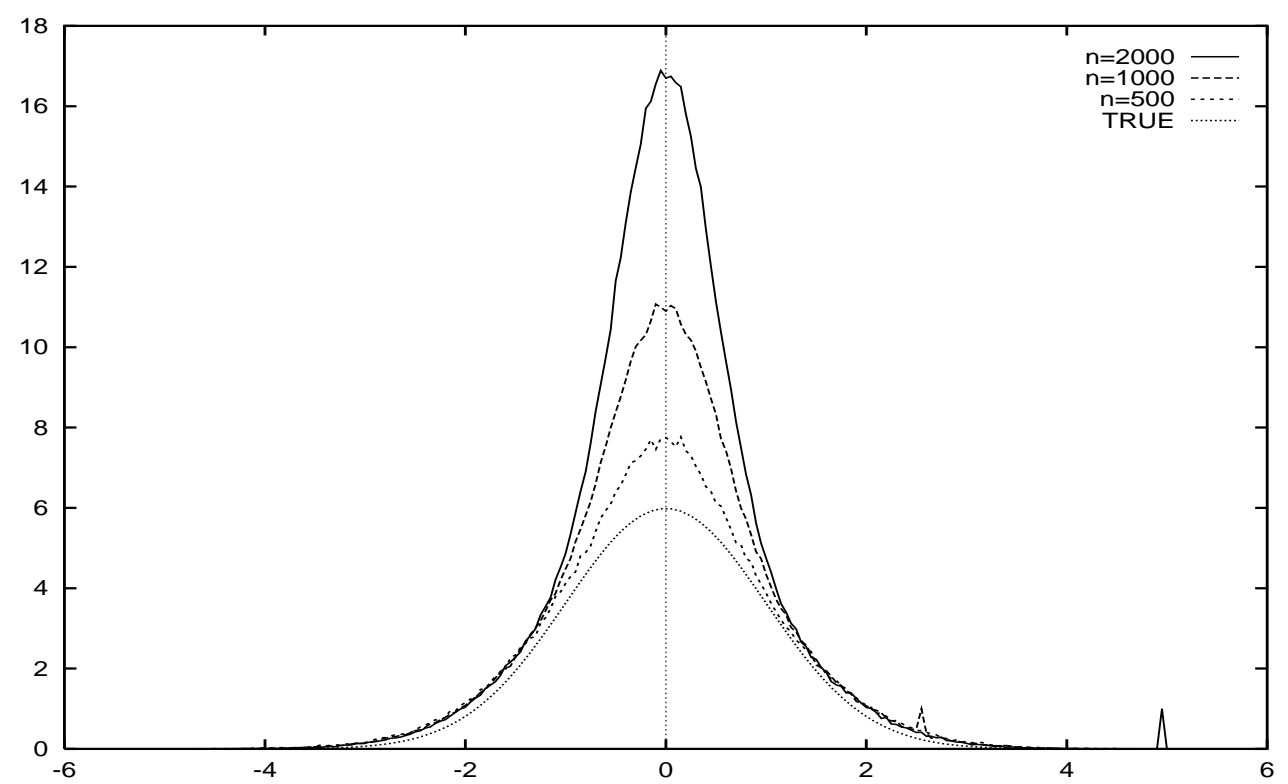

Figure 3. Estimation of the Lévy density in the model $f(z)=\lambda(\sqrt{2 \pi \nu})^{-1} \exp \left(-(2 \nu)^{-1} z^{2}\right)$ with $(\mu, \sigma, \lambda, \nu)=(-0.01,0.5,15.0,1.0), \rho=0.49, L=1.0$ for sample size 500,1000 and 2000, respectively.

From the intuitive point of view as above, it is better to choose $L$ at which the derivative is nearly zero visually. In fact, by evaluating $E\left[\hat{\lambda}_{n}(L)\right]$ analytically, we find that such derivatives can approximately decrease in the same way as the tail of the Lévy density $f(z)$ after that the filters exclude the influences by Brownian shocks: $\frac{d}{d L} E\left[\hat{\lambda}_{n}(L)\right] \approx 2 e^{-\lambda h_{n}} f(L)$ for large $L$; see Shimizu (2005). Therefore the derivatives can not be zero generally. However, if $F(z)$ has the light tail as the normal density and $\lambda \ll n$, then the curve of the derivatives look like zero compared with the enormous influences by diffusions.

Remark 4.1. Though we calculated the $L$-pointwise sample mean of $\hat{\lambda}_{n}(L)$ based on 1000 simulations in Figure 4, we could not calculate such a sample mean from the real data which is obtained in one sample path. However, if $n$ is sufficiently large, then we can use the estimator $\hat{\lambda}_{n}(L)$ from one sample path instead of $E\left[\hat{\lambda}_{n}(L)\right]$ since $\left|\hat{\lambda}_{n}(L)-E\left[\hat{\lambda}_{n}(L)\right]\right| \rightarrow 0(n \rightarrow \infty)$ in probability for each $L$; see Shimizu (2005).

We can choose, for example, that $(n, L)=(500,1.2),(3000,1.6)$ and $(10000,1.7)$ in view of Figure 5 . The result is shown in Figure 7 . We also show the case with $(n, L)=(15000,1.7)$ in that figure for the purpose of reference. Though we might not point out the optimal $L$ exactly, the performances are dramatically improved compared with in Figure 3. It would be useful in some practical situations although this is completely intuitive method. However, the constant $L$ should be chosen from more statistical point of view. A suitable 


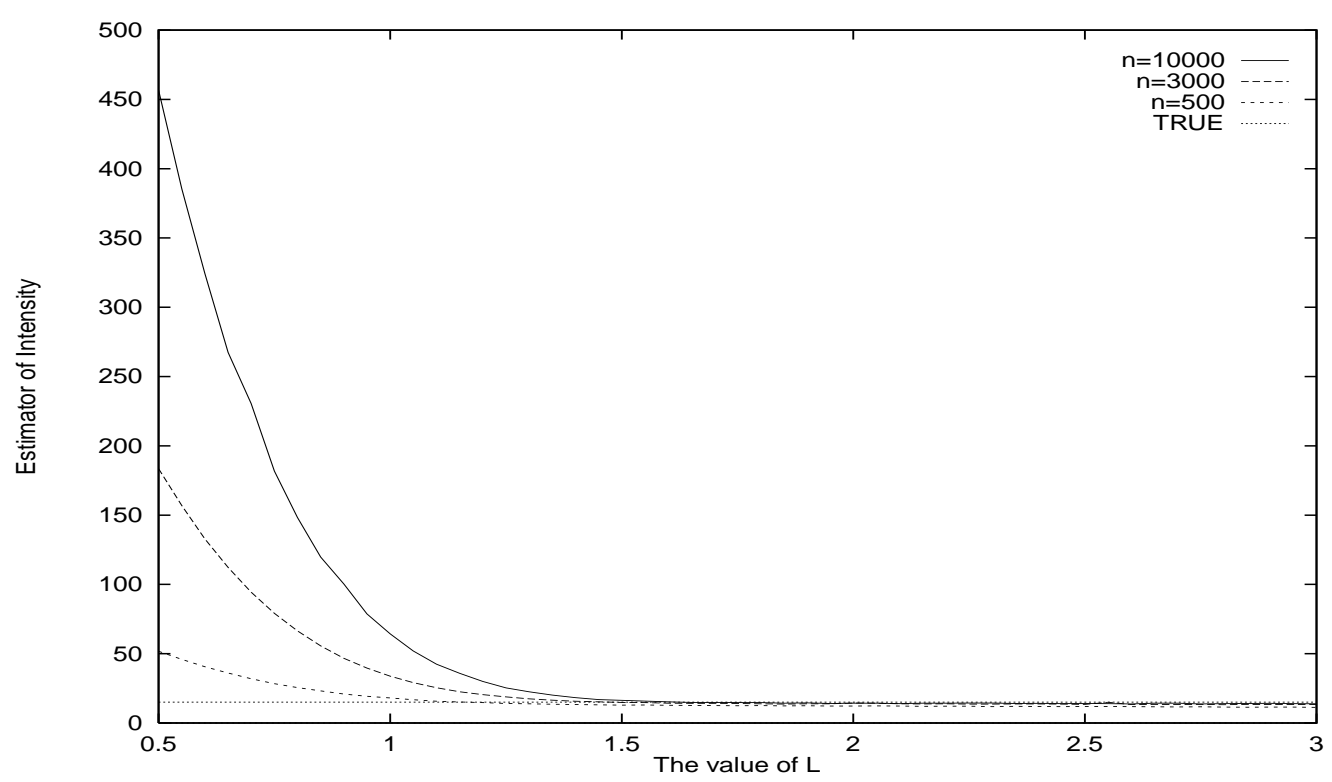

Figure 4. Estimation of the intensity $\lambda$ in the model $f(z)=\lambda(\sqrt{2 \pi \nu})^{-1} \exp \left(-(2 \nu)^{-1} z^{2}\right)$ with $(\mu, \sigma, \lambda, \nu)=(-0.01,0.5,15.0,1.0)$ for sample size 500,3000 and 10000 , respectively.

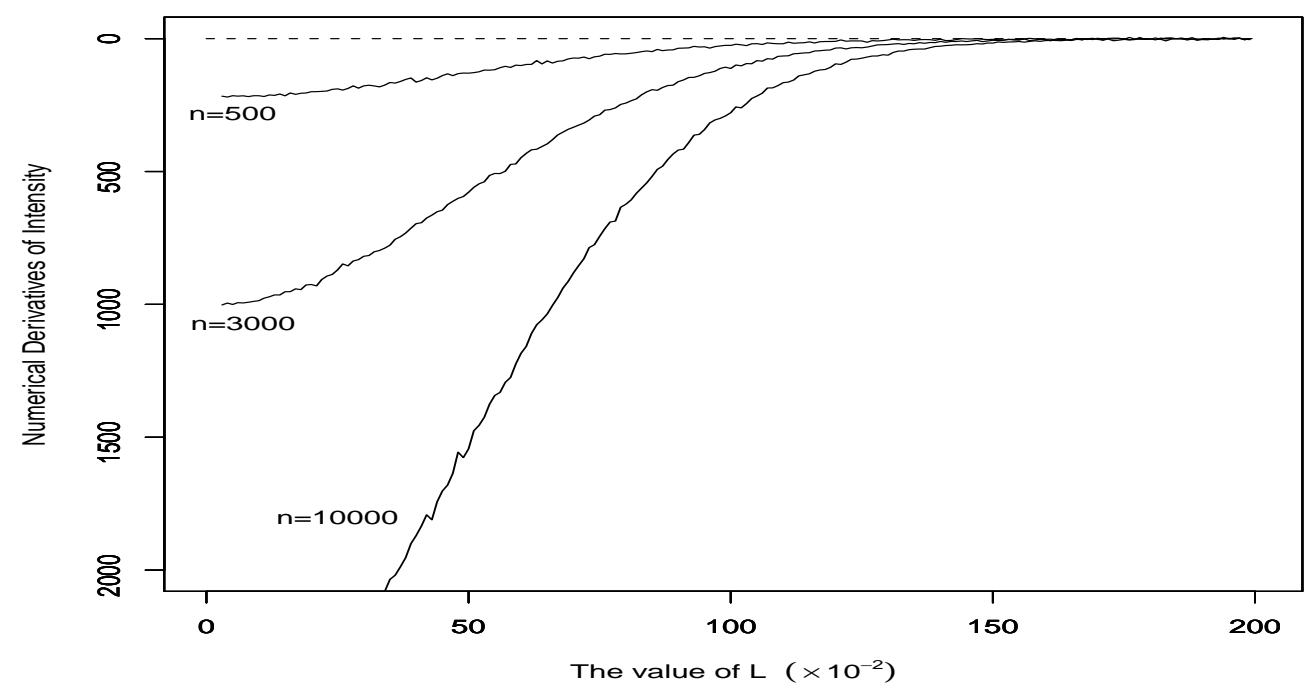

Figure 5. The five-points moving average curves of the numerical derivatives of $\hat{\lambda}_{n}(L)$ in $L$ for sample size 500, 3000 and 10000, respectively.

method has never been established yet, and it is a problem to be studied for the future.

For the purpose of reference, we reported the graphs of $\hat{\lambda}_{n}(L)$ for the model (4.2) in Figure 6. We will be able to guess that it would suffice to choose, for 


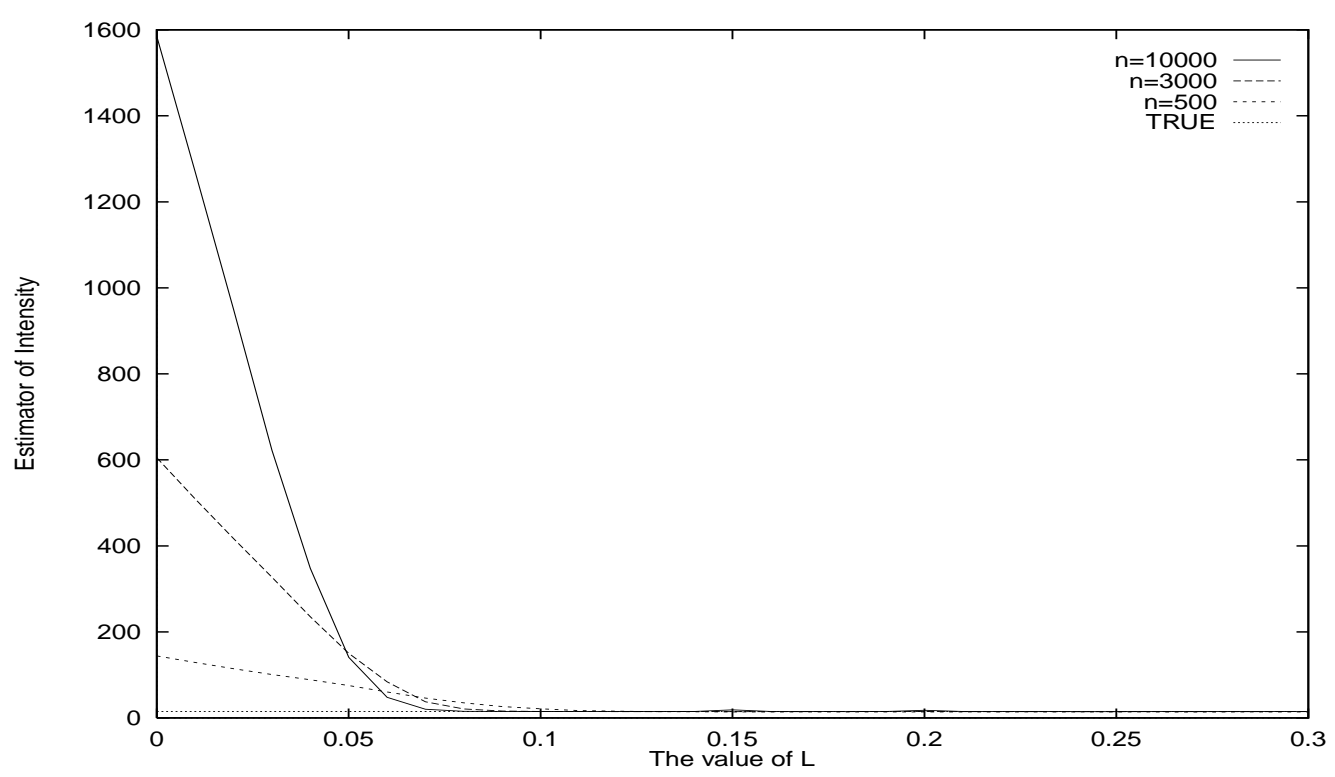

Figure 6. Estimation of the intensity $\lambda$ in the model $F(z)=2^{-1} \beta^{\alpha}|x|^{\alpha-1} \Gamma^{-1}(\alpha) \exp (-\beta|x|)$ with $(\mu, \sigma, \lambda, \alpha, \beta)=(-0.03,0.01,15.0,4.0,5.0)$ for sample size 500, 3000 and 10000 , respectively.

example, $0.15 \leq L \leq 0.2$ for each sample size. As already mentioned, $L=0.2$ was enough.

The view of Figure 6 also shows that our filters work asymptotically well for much smaller $L$ than 0.2 . Look at the curves around the interval [0.06,0.07] for each $n$. The intensity for $n=3000$ is estimated larger than for $n=500$ by some misjudgment, however, it appears to converge to the true value as $n=10000$. This shows that the consistency is true for the different selection of $L$. In the example of Figure 4, we could not show such phenomenon visually because of the too slow convergence of estimator. Intuitively speaking, the probability of misjudgment of jumps is about

$$
p_{n}:=P\left\{\left|\sigma W_{h_{n}}\right|>L h_{n}^{\rho}\right\}=2\left[1-\Phi\left(\sigma^{-1} L h_{n}^{\rho-1 / 2}\right)\right], \quad \rho \in[0,1 / 2) .
$$

In the setting of Figure $4, p_{10000}=0.031326$ and $p_{15000}=0.031318$, therefore the misjudgment is hardly improved at all. We would need to choose the sample size $n$ enormously large to demonstrate the consistency of $f_{n}$ regardless of the selection of $L$ in that setting, and it would exceed the ability of the usual calculator. However it must be possible to obtain the same result as in Figure 6 .

Now, we have to notice that such method of choosing $L$ is available when $X$ is ergodic. In the non-ergodic case, the estimator $\hat{\lambda}_{n}(L)$ might not converge even for large $L$ as in Figures 4 and 6. However, if the diffusion coefficient is known, we can modify the filters, and they can work well in the non-ergodic case. We discuss it in the next section. 


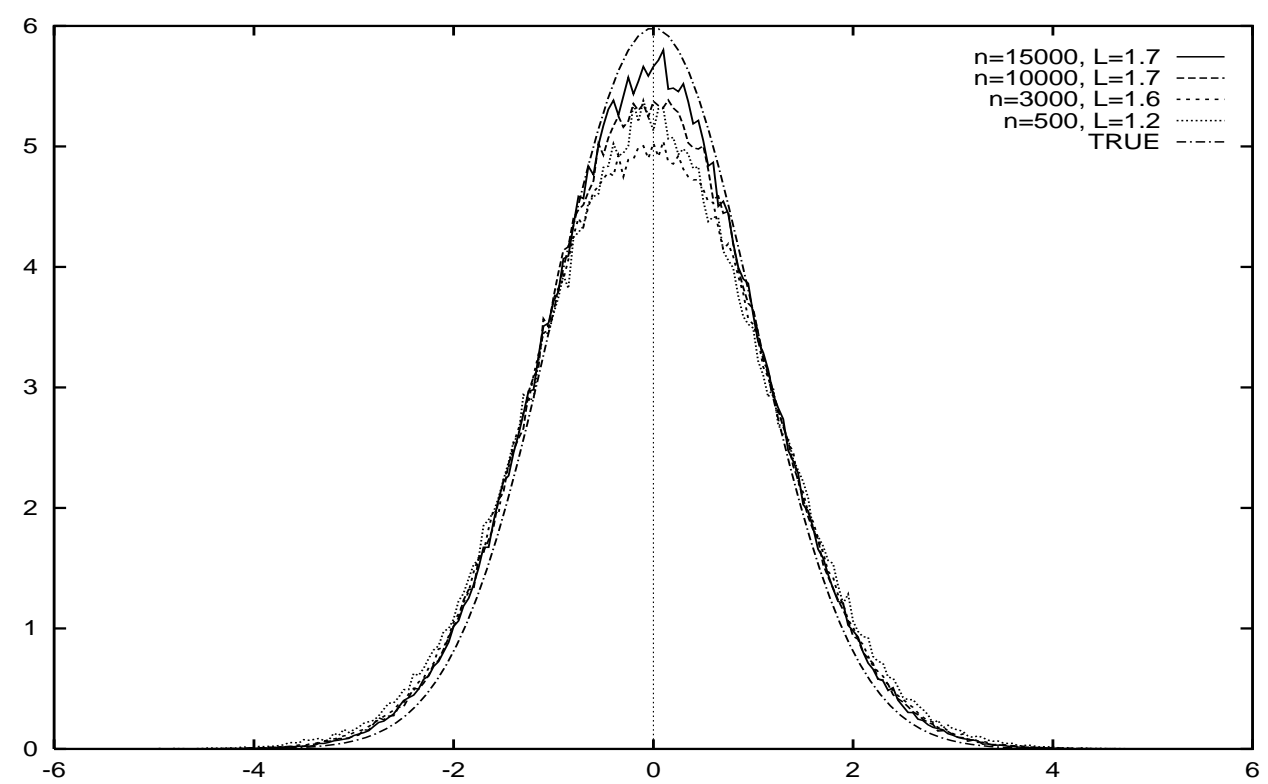

Figure 7. Estimation of the Lévy density in the model $f(z)=\lambda(\sqrt{2 \pi \nu})^{-1} \exp \left(-(2 \nu)^{-1} z^{2}\right)$ with $(\mu, \sigma, \lambda, \nu)=(-0.01,0.5,15.0,1.0)$ for sample size 500,3000 and 10000 , respectively.

\subsection{Data adaptive filters}

In the previous section, we described the ergodic case. If the model is nonergodic, then it is not necessarily the case that $\hat{\lambda}_{n}(L)$ converges to the true $\lambda$. Particularly, when the diffusion coefficient is Markovian type where $b\left(X_{t}\right)$ strictly depends on $X_{t}$; for example $b(x)=\sigma x$, then some simulations show that the behavior of $\hat{\lambda}_{n}(L)$ with respect to $L$ is unstable. However, if the diffusion coefficient $b(x)$ is known, we can construct the data adaptive filters. In this section, we consider the special case where the coefficient $b$ is known.

One will be able to see by the discussion in Shimizu and Yoshida (2002) that it may be possible to replace a constant $L$ by $\mathcal{F}_{t}$-adapted process $L_{t}\left(h_{n}\right)$ which satisfies some moment conditions and $L_{t_{i-1}^{n}}\left(h_{n}\right)=O_{p}\left(h_{n}^{\rho}\right)$ as $n \rightarrow \infty$, and to make the filters $\left\{\left|\Delta X_{i}^{n}\right|>L_{t_{i-1}^{n}}\left(h_{n}\right)\right\}$.

Suppose that $b^{2}\left(X_{t_{i-1}^{n}}\right)>0$. Note that, in one-dimensional case,

$$
N_{i, n}:=\left(\Delta X_{i}^{n}-a\left(X_{t_{i-1}^{n}}\right) h_{n}\right)\left(b^{2}\left(X_{t_{i-1}^{n}}\right) h_{n}\right)^{-1 / 2}
$$

approximately follows the standard normal distribution, which satisfies for large $n$ that

$$
P\left\{\left|\frac{\Delta X_{i}^{n}-a\left(X_{t_{i-1}^{n}}\right) h_{n}}{\sqrt{b^{2}\left(X_{t_{i-1}^{n}}\right) h_{n}}}\right|>u_{\alpha_{n} / 2}\right\} \approx \alpha_{n} \approx P\left\{\frac{\left|\Delta X_{i}^{n}\right|}{\sqrt{b^{2}\left(X_{t_{i-1}^{n}}\right) h_{n}}}>u_{\alpha_{n} / 2}\right\},
$$

where $u_{z}$ is the $z$-percentile of the standard normal distribution. Therefore

$$
L_{t_{i-1}^{n}}=u_{\alpha_{n} / 2} \sqrt{b^{2}\left(X_{t_{i-1}^{n}}\right) h_{n}} \vee h_{n}^{\rho}
$$




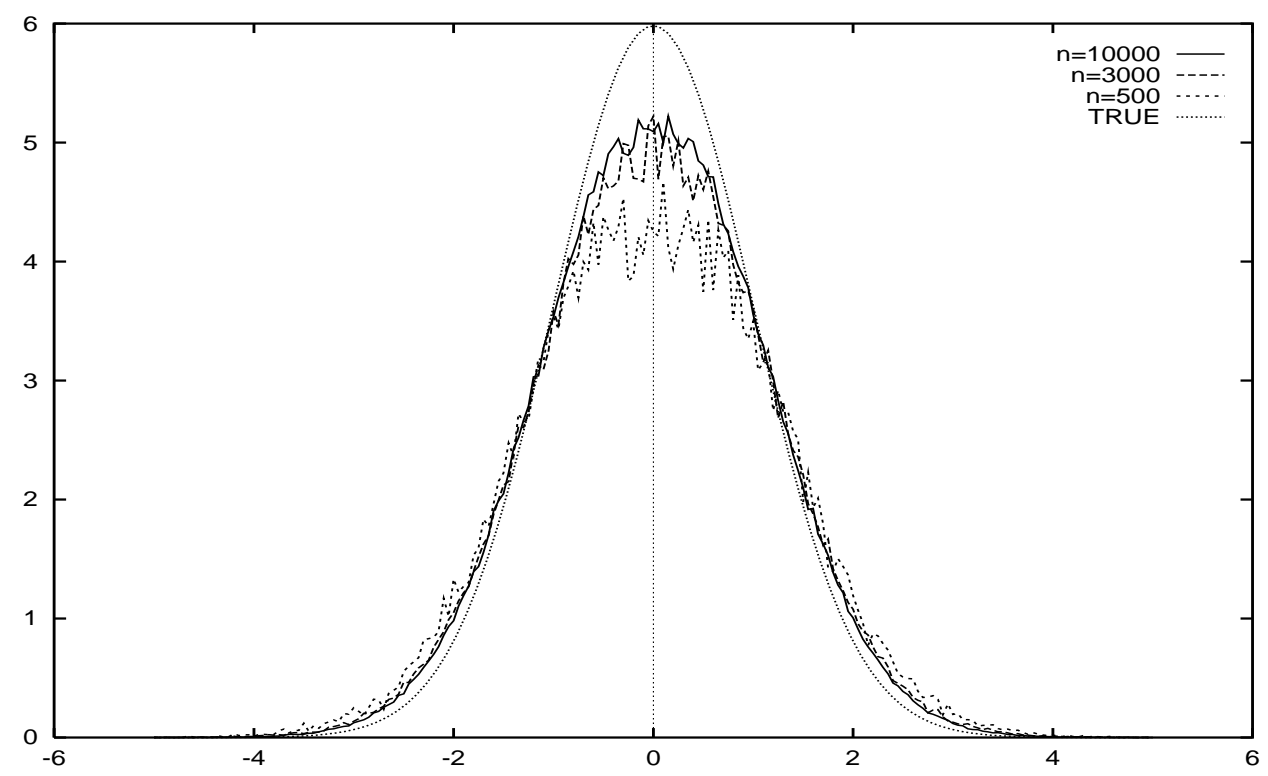

Figure 8. Estimation of the Lévy density in the model $d X_{t}=\mu X_{t} d t+\sigma_{0} X_{t} d W_{t}+d Z_{t}\left(\sigma_{0}=0.5\right.$ is known) and $f(z)=\lambda(\sqrt{2 \pi \nu})^{-1} \exp \left(-(2 \nu)^{-1} z^{2}\right)$ with $(\mu, \lambda, \nu)=(-0.01,15.0,1.0), \rho=0.49$ and $\alpha_{n} n=15$ for sample size 500, 3000 and 10000, respectively.

will be expected to improve the judgment of jumps. Here, we should choose the level $\alpha_{n}$ so that it depends on the observation number $n$ since the extent of misjudgments depends on $n$.

As an example of the non-ergodic case, let us consider the following model:

$$
d X_{t}=\mu X_{t} d t+\sigma_{0} X_{t} d W_{t}+d Z_{t},
$$

with the Lévy density $f(z)=\frac{\lambda}{\sqrt{2 \pi \nu}} \exp \left(-\frac{z^{2}}{2 \nu}\right)$ and $(\mu, \lambda, \nu)=(-0.01,15.0,1.0)$. We assume that $\sigma_{0}=0.5$ is known. We chose $\alpha_{n}$ as $\alpha_{n} n=15$ for $n=500$, 3000 , and 10000 . This implies that respectively $3 \%, 0.5 \%$, and $0.15 \%$ of the large shocks by diffusions are misjudged as jumps.

The estimation results are reported in Figure 8. It seems that the influences by diffusions are well excluded. However, it is not necessarily the case that $f_{n}$ becomes a good estimator as $n$ increases with $\alpha_{n} n=15$. The next Figure 9 shows the results of the case $100 \alpha_{n}=0.1(\%)$ and $0.15(\%)$ as $n=15000$. As $100 \alpha_{n}=0.15(\%)$, it seems that $f_{n}$ is a good estimator. On the other hand, as $100 \alpha_{n}=0.1(\%)\left(\alpha_{n} n=15\right)$, the filters cut too many small jumps, and $f_{n}$ underestimates around the origin.

Intuitively speaking, the mean of the number of misjudgments is roughly $e_{n}=\alpha_{n}\left(n-\lambda n h_{n}\right) \approx \alpha_{n} n$ for sufficiently large $n$ and $\lambda h_{n} \ll 1$, but the level $e_{n}$ should be chosen by an observer suitably so that those errors will not influence on estimation. However an observer could not determine the level without prior information. Therefore there remains room to consider how to choose the level $e_{n}$, and this should be done for the future. 


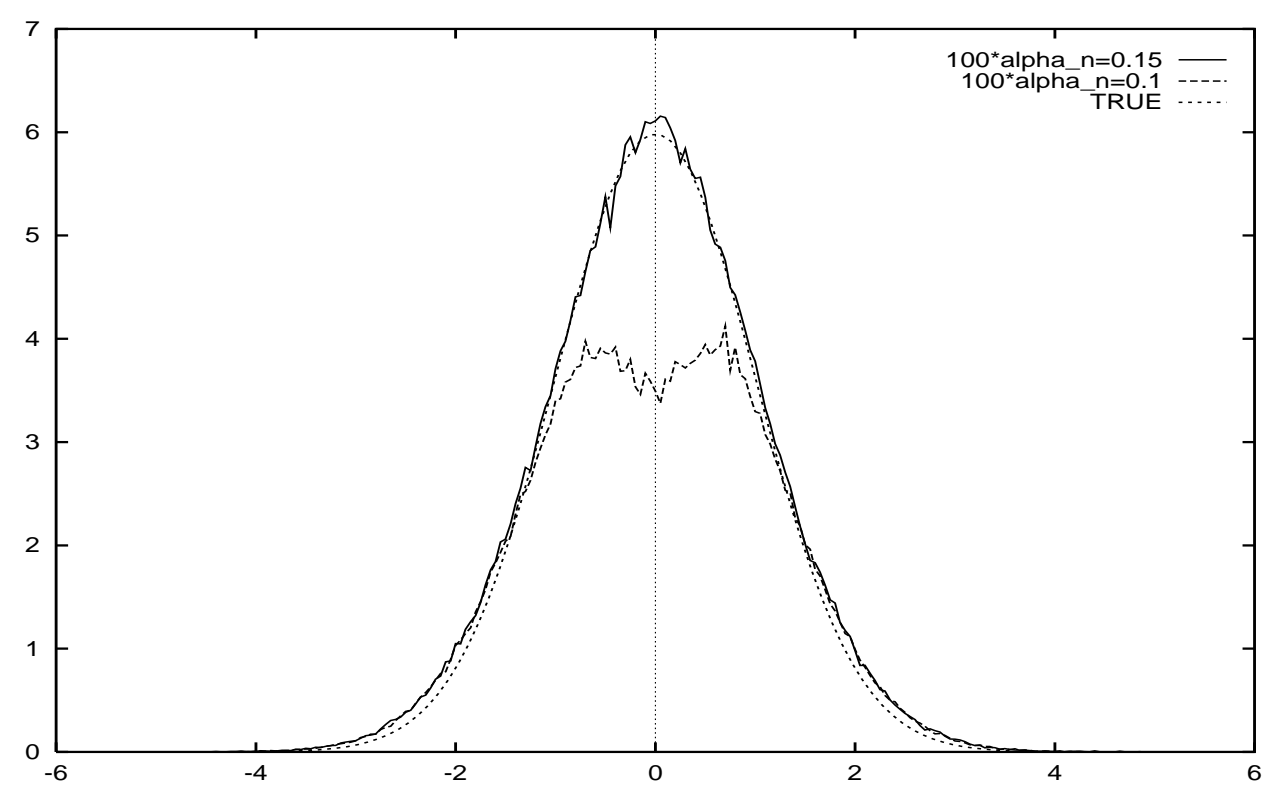

Figure 9. Estimation of the Lévy density in the model $d X_{t}=\mu X_{t} d t+\sigma_{0} X_{t} d W_{t}+d Z_{t}\left(\sigma_{0}=0.5\right.$ is known) and $f(z)=\lambda(\sqrt{2 \pi \nu})^{-1} \exp \left(-(2 \nu)^{-1} z^{2}\right)$ with $(\mu, \lambda, \nu)=(-0.01,15.0,1.0), \rho=0.49$ and $n=15000$ for $100 \alpha_{n}=0.15$ and $0.01(\%)$.

\section{Conclusion}

Recently, applications of stochastic processes with jumps have become more important than ever, and jump-diffusion models are one of frequently-used models. For that reason, the inference for diffusion processes with jumps is one of the most important problems. In particular, the inference from discrete observations is indispensable to applications.

This paper tackles the identification of Lévy density, which is the most important element to control the behavior of jumps, from the frequently observed data. Though we presented the inference from continuous observations in Theorem 3.1, this should be a benchmark for the inference from discrete observations, and it should certainly be considered before the inference from discrete observations.

One of the main contributions of this paper is that the filters to detect jumps enable us to estimate Lévy densities separately from discrete observations. We do not need to mind whether the continuous part is known or unknown, and whether the model is ergodic or non-ergodic, which is often important in the parametric setting. Therefore our theory can be applied to the wide class of the models in this sense. However there are some annoying restrictions on the order of $h_{n}$; the experimental design and $\delta_{n}$; the bandwidth: the conditions

$$
\begin{aligned}
& T_{n} h_{n}^{\nu}=O(1), \quad T_{n} \delta_{n}^{2 r+d}=O(1), \quad \delta_{n} h_{n}^{\rho-1 / 2}=O(1), \\
& T_{n} h_{n}^{1 / 2} \delta_{n}^{r-1}=o(1)
\end{aligned}
$$

should be satisfied as $n$ tends to infinity, where $T_{n}=n h_{n}, \nu \in\left(0, \frac{2 r+d}{2(2 r+d+1)}\right)$, 
$\rho \in\left[0, \frac{1}{2}\right), d$ is the dimension of the process and $r$ is the smoothness of Lévy densities; see Section 2.

The judgment of jumps contained in this paper is the existing treatment in Shimizu and Yoshida (2002), and we used the asymptotic filters $\left\{\left|\Delta X_{i}^{n}\right|>L h_{n}^{\rho}\right\}$ for a constant $L$. Though these filters asymptotically work well for arbitrary constant $L$ in theory, their capabilities actually depend on the constant $L$. Therefore, it is critical to choose $L$ under the practical situation that the number of observations $n$ is fixed.

What we can learn from the results of simulations is, although it is intuitively clear, that it is easy to identify the existence of jumps if the absolute value of the diffusion coefficient is relatively small compared with jump size. Actually, in two examples presented in Subsection 4.1, the estimator $f_{n}(z)$ does not diverge as long as we do not choose an $L$ which is extremely small. However it becomes difficult to choose $L$ when the diffusion noise as large as jump size occurs as in the example in Subsection 4.2, and it is possible that the asymptotic theory does not work well even under enormous sample size. Furthermore the statistical methodologies to select the suitable filters are uncertain and they are under consideration in Shimizu (2005).

Nevertheless, this paper tackles some intuitive methods to choose the constant $L$ from the data. The selection method of $L$ in Subsection 4.3 focuses attention on the difference between the order of the frequency of continuous noise and that of discontinuous breaks. In consequence of many simulations, we at least find the following:

- In ergodic case:

- This method can find the suitable constant $L$.

- In non-ergodic case:

- If the diffusion coefficient is uniformly bounded on the state space, then this method can find the suitable constant $L$.

- If the diffusion coefficient is unbounded Markovian type as in Subsection 4.4 , it is difficult to choose $L$ because of the instability behavior of the index (4.3).

From the second consideration, the index $\hat{\lambda}_{n}(L)$ may converge to the true $\lambda$ even if the model is non-ergodic. In the last case, we can exclude the influence of diffusions by using the predictable $L_{t_{i-1}^{n}}$ as shown in (4.4) if the diffusion coefficient is known. However there remains a problem to determine $\alpha_{n}$; the rate of misjudgment.

The assumptions for Lévy densities in this paper is a little restrictive, that is, $f(z)$ is bounded and continuous. However it is important in practice to consider Lévy densities, which diverge at the origin and are not integrable. In this case, the construction of new filters becomes a major problem as well as the order of $\nu$ and $\delta_{n}$. 


\section{Proofs}

\subsection{The proof of Theorem 3.1}

The proof follows the usual way of decomposing the MSE into bias and variance components and to show that they converge with optimal rate or faster. Notice the following decomposition.

$$
E\left|f_{T}(z)-f(z)\right|^{2}=b_{T}^{2}(z)+V f_{T}(z),
$$

where $b_{T}(z)=E f_{T}(z)-f(z), V f_{T}(z)=E f_{T}^{2}(z)-\left\{E f_{T}(z)\right\}^{2}$, and $K_{\delta_{T}}(z-x)=$ $\frac{1}{\delta_{T}^{d}} K\left(\frac{z-x}{\delta_{T}}\right)$.

Note that $N_{t}-\lambda t$ is $\mathscr{F}_{t}$-martingale. It follows from Theorem 3.2(i) in Prakasa Rao (1990) that

$$
\begin{aligned}
E f_{T}(z) & =\frac{1}{T} E\left[\int_{0}^{T} K_{\delta_{T}}\left(z-\Delta Z_{t}\right) d N_{t}\right] \\
& =\frac{\lambda}{T} \int_{0}^{T} E\left[K_{\delta_{T}}\left(z-\Delta Z_{t}\right)|| \Delta Z_{t} \mid>0\right] d t \\
& =\frac{1}{\delta_{T}^{d}} \int_{\mathbb{R}^{d}} K\left(\frac{z-u}{\delta_{T}}\right) \lambda F(u) d u \\
& =\int_{\mathbb{R}^{d}} K(v) f\left(z-\delta_{T} v\right) d v .
\end{aligned}
$$

As $m \neq 0$, by the same argument as the proof of Theorem 4.1 in Bosq (1998), we easily obtain that

$$
\left|b_{T}(z)\right| \leq c_{(r, m)} \delta_{T}^{m+l}
$$

where $c_{(r, m)}=\sum_{j_{1}+\cdots+j_{d}=m} \frac{\ell}{j_{1} ! \cdots j_{d} !} \int_{\mathbb{R}^{d}}|u|^{l}\left|u_{1}\right|^{j_{1}} \cdots\left|u_{d}\right|^{j_{d}}|K(u)| d u$.

Moreover, as $m=0$,

$$
\begin{aligned}
\left|b_{T}(z)\right| & \leq \int_{\mathbb{R}^{d}} K(v)\left|f\left(z-\delta_{T} v\right)-f(z)\right| d v \\
& \leq \ell \int_{\mathbb{R}^{d}} K(v)\left|\delta_{T} v\right|^{l} d v \\
& \leq c_{(r, 0)} \delta_{T}^{l} .
\end{aligned}
$$

Hence, for $m \geq 0$,

$$
T \delta_{T}^{d} b_{T}^{2}(z) \leq T \delta_{T}^{2 r+d} c_{(r, m)}^{2}=\eta_{T}^{2 r+d} c_{(r, m)}^{2} .
$$

Noticing $T \delta_{T}^{d}=T^{2 r /(2 r+d)} \eta_{T}^{d}$, we have $T^{2 r /(2 r+d)} b_{T}^{2}(z) \leq \eta_{T}^{2 r} c_{(r, m)}^{2}$.

The variance term $V f_{T}$ is dominated from above as follows:

$$
\begin{aligned}
V f_{T}(z) & \leq E\left|\frac{1}{T} \int_{0}^{T} K_{\delta_{T}}\left(z-\Delta Z_{t}\right) d N_{t}\right|^{2} \\
& =\frac{1}{T^{2} \delta_{T}^{d}} E\left[\int_{0}^{T} \frac{\lambda}{\delta_{T}^{d}} K^{2}\left(\frac{z-\Delta Z_{t}}{\delta_{T}}\right) d t|| \Delta Z_{t} \mid>0\right] \\
& \leq \frac{1}{T \delta_{T}^{d}} \sup _{z \in \mathbb{R}^{d}}|f(z)| \int_{\mathbb{R}^{d}} K^{2}(u) d u .
\end{aligned}
$$


This completes the proof.

\subsection{The proof of Theorem 3.2}

The proof is analogous to that of Theorem 3.1. We notice the following decomposition:

$$
\begin{aligned}
E\left|f_{n}(z)-f(z)\right|^{2} & =\left(E f_{n}(z)-f(z)\right)^{2}+E f_{n}^{2}(z)-\left(E f_{n}(z)\right)^{2} \\
& =: b_{n}^{2}+V f_{n},
\end{aligned}
$$

where $b_{n}(z)=E f_{n}(z)-f(z)$ and $V f_{n}(z)=E f_{n}^{2}(z)-\left(E f_{n}(z)\right)^{2}$. We define the stopping time $\tau_{i}^{n}$ as

$$
\tau_{i}^{n}:=\inf \left\{t \in\left[t_{i-1}^{n}, t_{i}^{n}\right) ;\left|\Delta Z_{t}\right|>0\right\},
$$

and let $b_{n}=b_{n}^{(1)}+b_{n}^{(2)}+b_{n}^{(3)}$, where

$$
\begin{aligned}
& b_{n}^{(1)}(z)=E f_{n}(z)-\frac{1}{T_{n}} \sum_{i=1}^{n} E\left[K_{\delta_{n}}\left(z-\Delta Z_{\tau_{i}^{n}}\right) \mathbf{1}_{\left\{J_{i}^{n}=1\right\}}\right] \\
& b_{n}^{(2)}(z)=\frac{1}{T_{n}} \sum_{i=1}^{n} E\left[K_{\delta_{n}}\left(z-\Delta Z_{\tau_{i}^{n}}\right) \mathbf{1}_{\left\{J_{i}^{n}=1\right\}}\right]-\frac{1}{T_{n}} \sum_{i=1}^{n} \lambda^{-1} f(z) P\left\{J_{i}^{n}=1\right\} \\
& b_{n}^{(3)}(z)=\frac{1}{T_{n}} \sum_{i=1}^{n} \lambda^{-1} f(z) P\left\{J_{i}^{n}=1\right\}-f(z) .
\end{aligned}
$$

On $b_{n}^{(1)}(z)$, applying Lemma A.2 with $p=1$, we have

$$
\begin{aligned}
\sup _{z \in \mathbb{R}^{d}}\left|b_{n}^{(1)}(z)\right| \leq & \frac{1}{T_{n} \delta_{n}^{d}} \sum_{i=1}^{n} \sup _{z \in \mathbb{R}^{d}} E\left[\mid k_{n}\left(z ; \Delta Z_{i}^{n}\right) \mathbf{1}_{\left\{\left|\Delta X_{i}^{n}\right|>L h_{n}^{\rho}\right\}}\right. \\
& \left.-k_{n}\left(z ; \Delta Z_{\tau_{i}^{n}}\right) \mathbf{1}_{\left\{J_{i}^{n}=1\right\}} \mid\right] \\
& \leq \frac{1}{T_{n} \delta_{n}^{d}} \sum_{j=1}^{n} \sum_{r=1}^{3} \sup _{z \in \mathbb{R}^{d}} E\left|K_{i, n}^{(r)}(z)\right| \\
& =\sqrt{h_{n}} \delta_{n}^{-(d+1)}\left\{O(1)+O\left(\delta_{n} h_{n}^{\rho-1 / 2}\right)\right\}=O\left(\sqrt{h_{n}} \delta_{n}^{-(d+1)}\right),
\end{aligned}
$$

where $k_{n}$ and $K_{i, n}^{(r)}(r=1,2,3)$ are given in Appendix. Therefore

$$
T_{n} \delta_{n}^{d} \sup _{z \in \mathbb{R}^{d}}\left\{b_{n}^{(1)}\right\}^{2}=O\left(\eta_{n}^{-(2 r+d)}\left(T_{n} h_{n}^{1 / 2} \delta_{n}^{r-1}\right)^{2}\right) \rightarrow 0 .
$$

On $b_{n}^{(2)}(z)$,

$$
\begin{aligned}
b_{n}^{(2)}(z)= & \frac{1}{T_{n}} \sum_{i=1}^{n} \int_{\mathbb{R}^{d}} K_{\delta_{n}}(z-u) \lambda^{-1} f(u) d u \times P\left\{J_{i}^{n}=1\right\} \\
& -\frac{1}{T_{n}} \sum_{i=1}^{n} \lambda^{-1} f(z) P\left\{J_{i}^{n}=1\right\}
\end{aligned}
$$




$$
\begin{aligned}
& =\frac{1}{T_{n}} \sum_{i=1}^{n} h_{n} e^{-\lambda h_{n}}\left\{\int_{\mathbb{R}^{d}} K_{\delta_{n}}(z-u) f(u) d u-f(z)\right\} \\
& =e^{-\lambda h_{n}} \int_{\mathbb{R}^{d}} K(v)\left\{f\left(z-\delta_{n} v\right)-f(z)\right\} d v .
\end{aligned}
$$

As $m \neq 0$, applying Taylor's formula to $f$,

$$
b_{n}^{(2)}(z)=e^{-\lambda h_{n}} \delta_{n}^{m} \int_{\mathbb{R}^{d}} K(v) \sum_{j_{1}+\cdots+j_{d}=m} \frac{v_{1}^{j_{1}} \cdots v_{d}^{j_{d}}}{j_{1} ! \cdots j_{d} !} \frac{\partial f^{(m)}}{\partial z_{1}^{j_{1}} \cdots \partial z_{d}^{j_{d}}}\left(z-\theta \delta_{n} v\right) d v,
$$

where $0<\theta<1$. Hence, by the same argument as in the proof of theorem 4.1 in Bosq (1998),

$$
T_{n} \delta_{n}^{d} \sup _{z \in \mathbb{R}^{d}}\left\{b_{n}^{(2)}(z)\right\}^{2} \leq \eta_{n}^{2 r+d} \eta_{(r, m)}^{2}
$$

where $\eta_{(r, m)}=\sum_{j_{1}+\cdots+j_{d}=m} \frac{\ell}{j_{1} ! \cdots j_{d} !} \int_{\mathbb{R}^{d}}|u|^{l}\left|u_{1}\right|^{j_{1}} \cdots\left|u_{d}\right|^{j_{d}}|K(u)| d u$. Moreover, as $m=0$, we can obtain (6.2) with $m=0$ by the same argument as in the proof of Theorem 3.1.

On $b_{n}^{(3)}(z)$,

$$
\begin{aligned}
\sup _{z \in \mathbb{R}^{d}}\left|b_{n}^{(3)}(z)\right| & =\sup _{z \in \mathbb{R}^{d}}\left|\frac{1}{T_{n}} \sum_{i=1}^{n} h_{n} e^{-\lambda h_{n}} f(z)-f(z)\right| \\
& =\sup _{z \in \mathbb{R}^{d}}\left|f(z) \| e^{-\lambda h_{n}}-1\right| \\
& =O\left(h_{n}\right) .
\end{aligned}
$$

This means

$$
T_{n} \delta_{n}^{d} \sup _{z \in \mathbb{R}^{d}}\left\{b_{n}^{(3)}(z)\right\}^{2}=O\left(T_{n} \delta_{n}^{d} h_{n}^{2}\right)=O\left(\left(T_{n} h_{n}^{\nu}\right) h_{n}^{2-\nu} \delta_{n}^{d}\right) \rightarrow 0
$$

Clearly, the quadratic cross terms from $b_{n}^{(1)}$ to $b_{n}^{(3)}$ converge to zero at rate $T_{n} \delta_{n}^{d}$ uniformly in $z$. Hence,

$$
\limsup _{n \rightarrow \infty} T_{n}^{2 r /(2 r+d)} \sup _{z \in \mathbb{R}^{d}} b_{n}^{2}(z) \leq \eta^{2 r} \cdot \eta_{(r)}
$$

Next, let us consider the term $V f_{n}$. We can decompose $V f_{n}$ as $V f_{n}:=$ $V_{n}+C_{n}$, where

$$
\begin{aligned}
& V_{n}(z)=\frac{1}{T_{n}^{2}} \sum_{i=1}^{n} \operatorname{Var}\left(K_{\delta_{n}}\left(z-\Delta Z_{i}^{n}\right)\right) \mathbf{1}_{\left\{\left|\Delta X_{i}^{n}\right|>L h_{n}^{\rho}\right\}}, \\
& C_{n}(z)=\frac{1}{T_{n}^{2}} \sum_{1 \leq i \neq j \leq n} \operatorname{Cov}\left(K_{\delta_{n}}\left(z-\Delta Z_{i}^{n}\right) \mathbf{1}_{\left\{\left|\Delta X_{i}^{n}\right|>L h_{n}^{\rho}\right\}},\right. \\
& \left.K_{\delta_{n}}\left(z-\Delta Z_{j}^{n}\right) \mathbf{1}_{\left\{\left|\Delta X_{j}^{n}\right|>L h_{n}^{\rho}\right\}}\right) .
\end{aligned}
$$


On $V_{n}(z)$, applying Lemma A.3, we have

$$
\begin{aligned}
V_{n}(z)= & \frac{1}{T_{n}^{2}} \sum_{i=1}^{n}\left\{E\left[K_{\delta_{n}}^{2}\left(z-\Delta Z_{i}^{n}\right) \mathbf{1}_{\left\{\left|\Delta X_{i}^{n}\right|>L h_{n}^{\rho}\right\}}\right]\right. \\
& \left.\quad-\left(E\left[K_{\delta_{n}}\left(z-\Delta Z_{i}^{n}\right) \mathbf{1}_{\left\{\left|\Delta X_{i}^{n}\right|>L h_{n}^{\rho}\right\}}\right]\right)^{2}\right\} \\
= & \frac{1}{T_{n} \delta_{n}^{d}}\left(g_{n, 2}(z)+C h_{n}^{1 / 2} \delta_{n}^{-(d+1)}\right),
\end{aligned}
$$

where $g_{n, p}(p>0)$ is given in Appendix.

On $C_{n}(z)$, noticing that

$$
\begin{aligned}
\operatorname{Cov}( & \left.K_{\delta_{n}}\left(z-\Delta Z_{i}^{n}\right) \mathbf{1}_{\left\{\left|\Delta X_{i}^{n}\right|>L h_{n}^{\rho}\right\}}, K_{\delta_{n}}\left(z-\Delta Z_{j}^{n}\right) \mathbf{1}_{\left\{\left|\Delta X_{j}^{n}\right|>L h_{n}^{\rho}\right\}}\right) \\
= & E\left[K_{\delta_{n}}\left(z-\Delta Z_{i}^{n}\right) \mathbf{1}_{\left\{\left|\Delta X_{i}^{n}\right|>L h_{n}^{\rho}\right\}} K_{\delta_{n}}\left(z-\Delta Z_{j}^{n}\right) \mathbf{1}_{\left\{\left|\Delta X_{j}^{n}\right|>L h_{n}^{\rho}\right\}}\right] \\
& \quad-E\left[K_{\delta_{n}}\left(z-\Delta Z_{i}^{n}\right) \mathbf{1}_{\left\{\left|\Delta X_{i}^{n}\right|>L h_{n}^{\rho}\right\}}\right] E\left[K_{\delta_{n}}\left(z-\Delta Z_{j}^{n}\right) \mathbf{1}_{\left\{\left|\Delta X_{j}^{n}\right|>L h_{n}^{\rho}\right\}}\right],
\end{aligned}
$$

and applying Lemma A.5 to the first term and Lemma A.3 to the second term in the right-hand side, we easily obtain $\sup _{z \in \mathbb{R}^{d}}\left|C_{n}(z)\right|=O\left(h_{n}^{1 / 2-\mu} \delta_{n}^{-(d+1+\mu d)}\right)$ for arbitrary $\mu \in(0,1)$. Hence,

$$
\begin{aligned}
T_{n} \delta_{n}^{d} \sup _{z \in \mathbb{R}^{d}} V f_{n}(z)= & \sup _{z \in \mathbb{R}^{d}} g_{n, 2}(z)+O\left(h_{n}^{1 / 2} \delta_{n}^{-(d+1)}\right)+O\left(T_{n} h_{n}^{1 / 2-\mu} \delta_{n}^{-1-\mu d}\right) \\
= & \sup _{z \in \mathbb{R}^{d}} g_{n, 2}(z)+O\left(\eta_{n}^{-(2 r+d)} \delta_{n}^{2 r-1} T_{n} h_{n}^{1 / 2}\right) \\
& +O\left(T_{n} h_{n}^{1 / 2-\mu} T_{n}^{(1+\mu d) /(2 r+d)}\right) \\
\leq & \sup _{z \in \mathbb{R}^{d}}|f(z)| \int K^{2}(u) d u+O\left(\delta_{n}^{2 r-1} T_{n} h_{n}^{1 / 2}\right) \\
& +O\left(T_{n}^{(2 r+d+1+\mu d) /(2 r+d)} h_{n}^{1 / 2-\mu}\right) .
\end{aligned}
$$

The last two terms in the last right-hand side tends to zero if we take $\mu \in(0,1)$ arbitrary small. Actually, $\delta_{n}^{2 r-1} T_{n} h_{n}^{1 / 2}=\left(T_{n} h_{n}^{1 / 2} \delta_{n}^{r-1}\right) \delta_{n}^{r} \rightarrow 0$ and $T_{n}^{(2 r+d+1+\mu d) /(2 r+d)} h_{n}^{1 / 2-\mu}=\left(T_{n} h_{n}^{\nu}\right)^{(2 r+d+1+\mu d) /(2 r+d)} h_{n}^{1 / 2-(2 r+d+1+\mu d) /(2 r+d) \nu-\mu}$ whose last index $\frac{1}{2}-\frac{2 r+d+1+\mu d}{2 r+d} \nu-\mu$ can be positive if we take $\mu$ sufficiently small since $0<\nu<\frac{2 r+d}{2(2 r+d+1)}$. As a result, we have

$$
T_{n} \delta_{n}^{d} \sup _{z \in \mathbb{R}^{d}} V f_{n}(z) \leq \sup _{z \in \mathbb{R}^{d}}|f(z)| \int K^{2}(u) d u+o\left(T_{n} h_{n}^{\nu}\right) .
$$

This completes the proof.

\section{Appendix A}

In this section, we make the same assumptions as in Theorem 3.2, and we use the following notations: for fixed $z \in \mathbb{R}^{d}$ and $q>0$,

$$
k_{n}(z ; x):=K\left(\frac{z-x}{\delta_{n}}\right), \quad g_{n, q}(z):=\int_{\mathbb{R}^{d}} \frac{1}{\delta_{n}^{d}} K^{q}\left(\frac{z-u}{\delta_{n}}\right) f(u) d u
$$




$$
\begin{aligned}
& K_{i, n, q}^{(1)}(z):=k_{n}^{q}\left(z ; \Delta Z_{i}^{n}\right) \mathbf{1}_{\left\{\left|\Delta X_{i}^{n}\right|>L h_{n}^{\rho}\right\}}-k_{n}^{q}\left(z ; \Delta Z_{i}^{n}\right) \mathbf{1}_{D_{i, 1}^{n}}, \\
& K_{i, n, q}^{(2)}(z):=k_{n}^{q}\left(z ; \Delta Z_{i}^{n}\right) \mathbf{1}_{D_{i, 1}^{n}}-k_{n}^{q}\left(z ; \Delta Z_{\tau_{i}^{n}}\right) \mathbf{1}_{D_{i, 1}^{n},}, \\
& K_{i, n, q}^{(3)}(z):=k_{n}^{q}\left(z ; \Delta Z_{\tau_{i}^{n}}\right) \mathbf{1}_{D_{i, 1}^{n}}-k_{n}^{q}\left(z ; \Delta Z_{\tau_{i}^{n}}\right) \mathbf{1}_{\left\{J_{i}^{n}=1\right\}},
\end{aligned}
$$

where $\tau_{i}^{n}$ is given in (6.1). Moreover we simply write $K_{i, n}^{(j)}(z)$ for $K_{i, n, 1}^{(j)}(z)$.

LEMmA A.1. Let $g(x)$ be a function of polynomial growth in $x$ defined on $\mathbb{R}^{k}$. Then

$$
E\left[\left|g\left(X_{t}\right)\right| \mid \mathscr{F}_{i-1}^{n}\right] \leq C\left(1+\left|X_{t_{i-1}^{n}}\right|\right)^{C},
$$

for any $t \in\left[t_{i-1}^{n}, t_{i}^{n}\right]$.

Proof. See Proposition 3.1 in Shimizu and Yoshida (2002).

Lemma A.2. For any $p, q \geq 1$,

$$
\begin{aligned}
& E\left[\left|K_{i, n, q}^{(1)}(z)\right|^{p} \mid \mathscr{F}_{i-1}^{n}\right]=R\left(z, h_{n}^{2}, X_{t_{i-1}^{n}}\right), \\
& E\left[\left|K_{i, n, q}^{(2)}(z)\right|^{p} \mid \mathscr{F}_{i-1}^{n}\right]=R\left(z, h_{n}^{p / 2+1} \delta_{n}^{-p}, X_{t_{i-1}^{n}}\right), \\
& E\left[\left|K_{i, n, q}^{(3)}(z)\right|^{p} \mid \mathscr{F}_{i-1}^{n}\right]=R\left(z, h_{n}^{\rho+1}, X_{t_{i-1}^{n}}\right) .
\end{aligned}
$$

Proof. On the equation (A.1), noticing that $k_{n}$ is bounded, we have

$$
\begin{aligned}
E\left[\left|K_{i, n, q}^{(1)}(z)\right|^{p} \mid \mathscr{F}_{i-1}^{n}\right] & \leq C_{p, q} E\left[\left|\mathbf{1}_{D_{i, 0}^{n}}+\mathbf{1}_{D_{i, 2}^{n}}\right|^{p} \mid \mathscr{F}_{i-1}^{n}\right] \\
& \leq C_{p, q}\left(P\left\{D_{i, 0}^{n} \mid \mathscr{F}_{i-1}^{n}\right\}+P\left\{D_{i, 2}^{n} \mid \mathscr{F}_{i-1}^{n}\right\}\right) \\
& =R\left(z, h_{n}^{2}, X_{t_{i-1}^{n}}\right) .
\end{aligned}
$$

The equation (A.3) is also similarly proved by using Lemma 3.1, that is,

$$
E\left[\left|K_{i, n, q}^{(3)}(z)\right|^{p} \mid \mathscr{F}_{i-1}^{n}\right] \leq C_{p, q} P\left\{C_{i, 1}^{n} \mid \mathscr{F}_{i-1}^{n}\right\}=R\left(z, h_{n}^{\rho+1}, X_{t_{i-1}^{n}}\right) .
$$

On the equation (A.2), applying the mean value theorem to $k_{n}^{q}$, we have

$$
\begin{aligned}
& E\left[\left|K_{i, n, q}^{(2)}(z)\right|^{p} \mid \mathscr{F}_{i-1}^{n}\right] \\
& \quad=E\left[\left|\delta_{n}^{-1} \partial_{x} k_{n}^{q}(z ; \tilde{Z})^{*} \partial_{y} c^{-1}\left(X_{\tau_{i}^{n}}, \Delta X_{\tau_{i}^{n}}\right)\right|^{p}\left|\Delta X_{i}^{n}-\Delta X_{\tau_{i}^{n}}\right|^{p} \mathbf{1}_{D_{i, 1}^{n}} \mid \mathscr{F}_{i-1}^{n}\right] \\
& \quad \leq C_{p, q} \delta_{n}^{-p} E\left[\left(\left|X_{t_{i}^{n}}-X_{\tau_{i}^{n}}\right|^{p}+\left|X_{\tau_{i}^{n}-}-X_{t_{i-1}^{n}}\right|^{p}\right) \mathbf{1}_{D_{i, 1}^{n}} \mid \mathscr{F}_{i-1}^{n}\right],
\end{aligned}
$$

where $\partial_{x}:=\left(\partial_{x_{1}}, \ldots, \partial_{x_{d}}\right)^{*}$, and $*$ stands for the transpose.

Since

$$
\begin{aligned}
E\left[\left|X_{\tau_{i}^{n}-}-X_{t_{i-1}^{n}}\right|{ }^{p} \mathbf{1}_{D_{i, 1}^{n} \mid} \mid \mathscr{F}_{i-1}^{n}\right] & \leq E\left[\left|X_{\tau_{i}^{n}-}-X_{t_{i-1}^{n}}\right|^{p} \mathbf{1}_{\left\{J_{i}^{n}=1\right\}} \mid \mathscr{F}_{i-1}^{n}\right] \\
& =R\left(z, h_{n}^{p / 2+1}, X_{t_{i-1}^{n}}\right)
\end{aligned}
$$

by the same argument as for the evaluation of $I_{1}$ in the proof of Proposition 3.6 in Shimizu and Yoshida (2002), and similarly

$$
E\left[\left|X_{t_{i}^{n}}-X_{\tau_{i}^{n}}\right|^{p} \mathbf{1}_{D_{i, 1}^{n}} \mid \mathscr{F}_{i-1}^{n}\right]=R\left(z, h_{n}^{p / 2+1}, X_{t_{i-1}^{n}}\right),
$$


we obtain $E\left[\left|K_{i, n, q}^{(2)}(z)\right|^{p} \mid \mathscr{F}_{i-1}^{n}\right]=R\left(z, h_{n}^{p / 2+1} \delta_{n}^{-p}, X_{t_{i-1}^{n}}\right)$.

Lemma A.3. For any $p>0$,

$$
\begin{aligned}
& E\left[K_{\delta_{n}}^{p}\left(z-\Delta Z_{i}^{n}\right) \mathbf{1}_{\left\{\left|\Delta X_{i}^{n}\right|>L h_{n}^{\rho}\right\}} \mid \mathscr{F}_{i-1}^{n}\right] \\
& \quad=h_{n} e^{-\lambda h_{n}} \delta_{n}^{-d(p-1)} g_{n, p}(z)+R\left(z, h_{n}^{3 / 2} \delta_{n}^{-(p d+1)}, X_{t_{i-1}^{n}}\right) .
\end{aligned}
$$

Proof. Notice that

$$
\begin{aligned}
E\left[K_{\delta_{n}}^{p}\left(z-\Delta Z_{i}^{n}\right) \mathbf{1}_{\left\{\left|\Delta X_{i}^{n}\right|>L h_{n}^{\rho}\right\}} \mid \mathscr{F}_{i-1}^{n}\right] \\
=\delta_{n}^{-p d}\left\{E\left[K_{i, n, p}^{(1)}(z) \mid \mathscr{F}_{i-1}^{n}\right]+E\left[K_{i, n, p}^{(2)}(z) \mid \mathscr{F}_{i-1}^{n}\right]+E\left[K_{i, n, p}^{(3)}(z) \mid \mathscr{F}_{i-1}^{n}\right]\right\} \\
\quad+E\left[\delta_{n}^{-p d} k_{n}^{p}\left(z ; \Delta Z_{\tau_{i}^{n}}\right) \mathbf{1}_{\left\{J_{i}^{n}=1\right\}} \mid \mathscr{F}_{i-1}^{n}\right] .
\end{aligned}
$$

Since $\left(\Delta Z_{\tau_{i}^{n}}, J_{i}^{n}\right)$ and $\mathscr{F}_{i-1}^{n}$ are independent for each $i$,

$$
\begin{aligned}
& E\left[K_{\delta_{n}}^{p}\left(z-\Delta Z_{i}^{n}\right) \mathbf{1}_{\left\{\left|\Delta X_{i}^{n}\right|>L h_{n}^{\rho}\right\}} \mid \mathscr{F}_{i-1}^{n}\right] \\
&=\delta_{n}^{-d(p-1)} E\left[E\left[\delta_{n}^{-d} k_{n}^{p}\left(s ; \Delta Z_{\tau_{i}^{n}}\right) \mid J_{i}^{n}=1\right] \mathbf{1}_{\left\{J_{i}^{n}=1\right\}} \mid \mathscr{F}_{i-1}^{n}\right] \\
& \quad+R\left(z, h_{n}^{3 / 2} \delta_{n}^{-(p d+1)}, X_{t_{i-1}^{n}}\right)\left(\sqrt{h_{n}} \delta_{n}+1+\delta_{n} h_{n}^{\rho-1 / 2}\right) \\
&=\delta_{n}^{-d(p-1)} P\left\{J_{i}^{n}=1\right\} \int_{\mathbb{R}^{d}} \frac{1}{\delta_{n}^{d}} K^{p}\left(\frac{z-u}{\delta_{n}}\right) \lambda^{-1} f(u) d u \\
&+R\left(z, h_{n}^{3 / 2} \delta_{n}^{-(p d+1)}, X_{t_{i-1}^{n}}\right)\left(\sqrt{h_{n}} \delta_{n}+1+\delta_{n} h_{n}^{\rho-1 / 2}\right) \\
&= h_{n} e^{-\lambda h_{n}} \delta_{n}^{-d(p-1)} g_{n, p}(z)+R\left(z, h_{n}^{3 / 2} \delta_{n}^{-(p d+1)}, X_{t_{i-1}^{n}}\right) .
\end{aligned}
$$

Remark A.1. We can easily obtain the following equalities by the same argument as above.

$$
\begin{aligned}
& E\left[K_{\delta_{n}}^{p}\left(z-\Delta Z_{i}^{n}\right) \mathbf{1}_{D_{i, 1}}^{n} \mid \mathscr{F}_{i-1}^{n}\right] \\
& \quad=h_{n} e^{-\lambda h_{n}} \delta_{n}^{-d(p-1)} g_{n, p}(z)+R\left(z, h_{n}^{3 / 2} \delta_{n}^{-(p d+1)}, X_{t_{i-1}^{n}}\right), \\
& E\left[K_{\delta_{n}}^{p}\left(z-\Delta Z_{\tau_{i}^{n}}\right) \mathbf{1}_{D_{i, 1}}^{n} \mid \mathscr{F}_{i-1}^{n}\right] \\
& \quad=h_{n} e^{-\lambda h_{n}} \delta_{n}^{-d(p-1)} g_{n, p}(z)+R\left(z, h_{n}^{1+\rho} \delta_{n}^{-p d}, X_{t_{i-1}^{n}}\right) .
\end{aligned}
$$

We shall omit these proofs.

LEMMA A.4. Let $Q(x)$ be a real valued function defined on $\mathbb{R}^{d}$ which is of polynomial growth. We set $Q_{j}:=Q\left(X_{j}^{n}\right)$. Then, for any $p>1$ and $i<j$,

(A.6) $\quad E\left[Q_{j-1} K_{\delta_{n}}\left(z-\Delta Z_{i}^{n}\right) \mathbf{1}_{\left\{\left|\Delta X_{i}^{n}\right|>L h_{n}^{\rho}\right\}} \mid \mathscr{F}_{i-1}^{n}\right]=R\left(z, h_{n}^{1 / p} \delta_{n}^{-d}, X_{i-1}^{n}\right)$,

(A.7) $E\left[Q_{j-1} K_{i, n}^{(1)}(z) \mid \mathscr{F}_{i-1}^{n}\right]=R\left(z, h_{n}^{2 / p}, X_{i-1}^{n}\right)$,

(A.8) $E\left[Q_{j-1} K_{i, n}^{(2)}(z) \mid \mathscr{F}_{i-1}^{n}\right]=R\left(z, h_{n}^{1 / 2+1 / p} \delta_{n}^{-1}, X_{i-1}^{n}\right)$,

(A.9) $E\left[Q_{j-1} K_{i, n}^{(3)}(z) \mid \mathscr{F}_{i-1}^{n}\right]=R\left(z, h_{n}^{(\rho+1) / p}, X_{i-1}^{n}\right)$. 
Proof. Notice that $E\left[Q_{j-1} \mid \mathscr{F}_{i-1}^{n}\right]=R\left(z, 1, X_{i-1}^{n}\right)$ by Lemma A.1. The straightforward calculations using Lemmas A.2, A.3 and Hölder inequality yield (A.6)-(A.9).

Lemma A.5. For $i<j$ and arbitrary $\mu \in(0,1)$,

$$
\begin{gathered}
E\left[K_{\delta_{n}}\left(z-\Delta Z_{i}^{n}\right) \mathbf{1}_{\left\{\left|\Delta X_{i}^{n}\right|>L h_{n}^{\rho}\right\}} K_{\delta_{n}}\left(z-\Delta Z_{j}^{n}\right) \mathbf{1}_{\left\{\left|\Delta X_{j}^{n}\right|>L h_{n}^{\rho}\right\}} \mid \mathscr{F}_{i-1}^{n}\right] \\
=h_{n}^{2}\left\{e^{-2 \lambda h_{n}} g_{n, 1}^{2}(z)+R\left(z, h_{n}^{1 / 2-\mu} \delta_{n}^{-(d+1+\mu d)}, X_{i-1}^{n}\right)\right\} .
\end{gathered}
$$

Proof. Since $\left(\Delta Z_{\tau_{i}^{n}}, J_{i}^{n}\right),\left(\Delta Z_{\tau_{j}^{n}}, J_{j}^{n}\right)$ and $\mathscr{F}_{i-1}^{n}$ are independent each other for $i<j$, we see that

$$
\begin{aligned}
M_{n} & :=E\left[K_{\delta_{n}}\left(z-\Delta Z_{\tau_{i}^{n}}\right) \mathbf{1}_{\left\{J_{i}^{n}=1\right\}} K_{\delta_{n}}\left(z-\Delta Z_{\tau_{j}^{n}}\right) \mathbf{1}_{\left\{J_{j}^{n}=1\right\}} \mid \mathscr{F}_{i-1}^{n}\right] \\
& =\left\{\int_{\mathbb{R}^{d}} \frac{1}{\delta_{n}^{d}} K\left(\frac{z-u}{\delta_{n}}\right) \lambda^{-1} f(u) d u \cdot \lambda h_{n} e^{-\lambda h_{n}}\right\}^{2} \\
& =h_{n}^{2} e^{-2 \lambda h_{n}} g_{n, 1}^{2}(z) .
\end{aligned}
$$

Let $E_{n}:=E\left[K_{\delta_{n}}\left(z-\Delta Z_{i}^{n}\right) \mathbf{1}_{\left\{\left|\Delta X_{i}^{n}\right|>L h_{n}^{\rho}\right\}} K_{\delta_{n}}\left(z-\Delta Z_{j}^{n}\right) \mathbf{1}_{\left\{\left|\Delta X_{j}^{n}\right|>L h_{n}^{\rho}\right\}} \mid \mathscr{F}_{i-1}^{n}\right]$.

$$
\begin{aligned}
\delta^{d}\left(E_{n}-M_{n}\right)= & E\left[K_{\delta_{n}}\left(z-\Delta Z_{i}^{n}\right) \mathbf{1}_{\left\{\left|\Delta X_{i}^{n}\right|>L h_{n}^{\rho}\right\}} E\left[K_{j, n}^{(1)}(z) \mid \mathscr{F}_{j-1}^{n}\right] \mid \mathscr{F}_{i-1}^{n}\right] \\
& +E\left[E\left[K_{\delta_{n}}\left(z-\Delta Z_{j}^{n}\right) \mathbf{1}_{D_{j, 1}^{n}} \mid \mathscr{F}_{j-1}^{n}\right] K_{i, n}^{(1)}(z) \mid \mathscr{F}_{i-1}^{n}\right] \\
& +E\left[K_{\delta_{n}}\left(z-\Delta Z_{i}^{n}\right) \mathbf{1}_{D_{i, 1}^{n}} E\left[K_{j, n}^{(2)}(z) \mid \mathscr{F}_{j-1}^{n}\right] \mid \mathscr{F}_{i-1}^{n}\right] \\
& +E\left[E\left[K_{\delta_{n}}\left(z-\Delta Z_{\tau_{j}^{n}}\right) \mathbf{1}_{D_{j, 1}^{n}} \mid \mathscr{F}_{j-1}^{n}\right] K_{i, n}^{(2)}(z) \mid \mathscr{F}_{i-1}^{n}\right] \\
& +E\left[K_{\delta_{n}}\left(z-\Delta Z_{\left.\tau_{i}^{n}\right)} \mathbf{1}_{D_{i, 1}^{n}} E\left[K_{j, n}^{(3)}(z) \mid \mathscr{F}_{j-1}^{n}\right] \mid \mathscr{F}_{i-1}^{n}\right]\right. \\
& +E\left[E\left[K_{\delta_{n}}\left(z-\Delta Z_{\tau_{j}^{n}}\right) \mathbf{1}_{\left\{J_{j}^{n}=1\right\}} \mid \mathscr{F}_{j-1}^{n}\right] K_{i, n}^{(3)}(z) \mid \mathscr{F}_{i-1}^{n}\right] .
\end{aligned}
$$

From (A.4) and (A.5) with $p=1$, we find that

$$
\begin{aligned}
& E\left[K_{\delta_{n}}\left(z-\Delta Z_{j}^{n}\right) \mathbf{1}_{D_{j, 1}^{n}} \mid \mathscr{F}_{j-1}^{n}\right]=R\left(z, h_{n}, X_{t_{j-1}^{n}}\right), \\
& E\left[K_{\delta_{n}}\left(z-\Delta Z_{\tau_{j}^{n}}\right) \mathbf{1}_{D_{j, 1}^{n}} \mid \mathscr{F}_{j-1}^{n}\right]=R\left(z, h_{n}, X_{t_{j-1}^{n}}\right), \\
& E\left[K_{\delta_{n}}\left(z-\Delta Z_{\tau_{j}^{n}}\right) \mathbf{1}_{\left\{J_{j}^{n}=1\right\}} \mid \mathscr{F}_{j-1}^{n}\right]=R\left(z, h_{n}, X_{t_{j-1}^{n}}\right)
\end{aligned}
$$

since $g_{n, 1}(z)$ is bounded by Bochner's lemma. Hence we obtain that

$$
\begin{aligned}
& \text { (A.10) } \quad E_{n}-M_{n}=E\left[K_{\delta_{n}}\left(z-\Delta Z_{i}^{n}\right) \mathbf{1}_{\left\{\left|\Delta X_{i}^{n}\right|>L h_{n}^{\rho}\right\}} R\left(z, h_{n}^{2} \delta_{n}^{-d}, X_{t_{j-1}^{n}}\right) \mid \mathscr{F}_{i-1}^{n}\right] \\
& +E\left[R\left(z, h_{n} \delta_{n}^{-d}, X_{t_{j-1}^{n}}\right) K_{i, n}^{(1)}(z) \mid \mathscr{F}_{i-1}^{n}\right] \\
& +E\left[K_{\delta_{n}}\left(z-\Delta Z_{i}^{n}\right) \mathbf{1}_{D_{i, 1}^{n}} R\left(z, h_{n}^{3 / 2} \delta_{n}^{-(d+1)}, X_{t_{j-1}^{n}}\right) \mid \mathscr{F}_{i-1}^{n}\right] \\
& +E\left[R\left(z, h_{n} \delta_{n}^{-d}, X_{t_{j-1}^{n}}\right) K_{i, n}^{(2)}(z) \mid \mathscr{F}_{i-1}^{n}\right] \\
& +E\left[K_{\delta_{n}}\left(z-\Delta Z_{\tau_{i}^{n}}\right) \mathbf{1}_{D_{i, 1}^{n}} R\left(z, h_{n}^{\rho+1} \delta_{n}^{-d}, X_{t_{j-1}^{n}}\right) \mid \mathscr{F}_{i-1}^{n}\right] \\
& +E\left[R\left(z, h_{n} \delta_{n}^{-d}, X_{t_{j-1}^{n}}\right) K_{i, n}^{(3)}(z) \mid \mathscr{F}_{i-1}^{n}\right] .
\end{aligned}
$$


On the term (A.12), using Hölder inequality and (A.4),

$$
\begin{aligned}
& E\left[K_{\delta_{n}}\left(z-\Delta Z_{i}^{n}\right) \mathbf{1}_{D_{i, 1}^{n}} R\left(z, h_{n}^{3 / 2} \delta_{n}^{-(d+1)}, X_{t_{j-1}^{n}}\right) \mid \mathscr{F}_{i-1}^{n}\right] \\
& \quad \leq C h_{n}^{3 / 2} \delta_{n}^{-(d+1)}\left\{E\left[K_{\delta_{n}}^{p}\left(z-\Delta Z_{i}^{n}\right) \mathbf{1}_{D_{i, 1}^{n}} \mid \mathscr{F}_{i-1}^{n}\right]\right\}^{1 / p} \\
& \quad=R\left(z, \alpha_{n}, X_{t_{j-1}^{n}}\right)
\end{aligned}
$$

for any $p>1$, where $\alpha_{n}=h_{n}^{3 / 2+1 / p} \delta_{n}^{-(d+1)-(p-1) d / p}$. Similarly, we can obtain that (A.10) and (A.14) are also $R\left(z, \alpha_{n}, X_{i-1}^{n}\right)$ since

$$
\begin{aligned}
& R\left(z, h_{n}^{2} \delta_{n}^{-d}, X_{t_{j-1}^{n}}\right)=\delta_{n} h_{n}^{1 / 2} R\left(z, h_{n}^{3 / 2} \delta_{n}^{-(d+1)}, X_{i-1}^{n}\right), \\
& R\left(z, h_{n}^{\rho+1} \delta_{n}^{-d}, X_{i-1}^{n}\right)=\delta_{n} h_{n}^{\rho-1 / 2} R\left(z, h_{n}^{3 / 2} \delta_{n}^{-(d+1)}, X_{i-1}^{n}\right) .
\end{aligned}
$$

Moreover we see that (A.13) is also $R\left(z, \alpha_{n}, X_{i-1}^{n}\right)$ by (A.8). Therefore, applying Lemma A.4 to (A.11) and (A.15), we have

$$
\begin{aligned}
E_{n}-M_{n} & =R\left(z, h_{n}^{1+2 / p} \delta_{n}^{-d}, X_{i-1}^{n}\right)+R\left(z, h_{n}^{1+(\rho+1) / p^{\prime}} \delta_{n}^{-d}, X_{i-1}^{n}\right)+R\left(z, \alpha_{n}, X_{i-1}^{n}\right) \\
& =\alpha_{n}\left\{R\left(z, h_{n}^{1 / p-1 / 2} \delta_{n}^{1+d-1 / p}, X_{t_{i-1}^{n}}\right)+R\left(z, \beta_{n}, X_{t_{i-1}^{n}}\right)+R\left(z, 1, X_{t_{i-1}^{n}}^{n}\right)\right\}
\end{aligned}
$$

for any $p, p^{\prime}>1$, where $\beta_{n}:=\alpha_{n}^{-1} h_{n}^{1+(\rho+1) / p^{\prime}} \delta_{n}^{-d}$. Let $1 / p=1-\mu$ and $(\rho+1) / p^{\prime}=$ $\rho+1-\mu^{\prime}$ for $\mu, \mu^{\prime} \in(0,1)$, then

$$
\beta_{n}=\left(h_{n}^{\rho-1 / 2} \delta_{n}\right) \cdot h_{n}^{\mu-\mu^{\prime}} \delta_{n}^{1+\mu d} .
$$

Hence, if we take $\mu \geq \mu^{\prime}$ then $\beta_{n} \rightarrow 0$. Consequently,

$$
\begin{aligned}
E_{n} & =M_{n}+R\left(z, \alpha_{n}, X_{i-1}^{n}\right) \\
& =M_{n}+h_{n}^{2} R\left(z, h_{n}^{1 / 2-\mu} \delta_{n}^{-(d+1+\mu d)}, X_{i-1}^{n}\right) .
\end{aligned}
$$

We can take $\mu \in(0,1)$ arbitrary since $\mu=1-1 / p$ for any $p>1$. This completes the proof.

\section{Acknowledgements}

The author extends his grateful thanks to the two anonymous referees for their helpful suggestions and exact corrections. Many thanks go to the editor for a lot of help. He is also grateful to Prof. N. Yoshida for his valuable comments and encouragement.

\section{REFERENCES}

Bandi, F. M. and Nguyen, T. H. (2003). On the functional estimation of jump-diffusion models, J. Econometrics, 116, 211-229.

Bosq, D. (1998). Nonparametric Statistics for Stochastic Processes, 2nd ed., Springer-Verlag, New York.

Cont, R. and Tankov, P. (2004). Financial Modeling with Jump Processes, Chapman \& Hall/CRC, Financial Mathematics Series.

Ibragimov, I. A. and Has'minskii, R. Z. (1981). Statistical Estimaion, Springer-Verlag, Berlin. 
Jacod, J. and Shiryaev, A. N. (1987). Limit Theorems for Stochastic Processes, Springer-Verlag, Berlin.

Kessler, M. (1997). Estimation of diffusion processes from discrete observations, Scand. J. Statist., 24, 211-229.

Masry, E. (1983). Probability density estimation from sampled data, IEEE Trans. Inform. Theory, 29, 696-709.

Masuda, H. (2004). Ergodicity and exponential $\beta$-mixing bounds for a strong solution of Lévydriven stochastic differential equations, MHF preprint series, Kyushu University, 2004-19.

Nguyen, H. T. and Pham, T. D. (1981). Nonparametric estimation in diffusion model by discrete sampling, Pub. Inst. Stat. Univ. Paris, XXVI, fasc. 2, 89-109.

Parzen, E. (1962). On the estimation of probability density function and mode, Ann. Math. Statist., 33, 1065-1076.

Prakasa Rao, B. L. S. (1988). Statistical inference from sampled data for stochastic processes, Statistical Inference from Stochastic Processes (Ithaca, NY, 1987), 249-284, Contemp. Math., 80, Amer. Math. Soc., Providence, RI.

Prakasa Rao, B. L. S. (1990). Nonparametric density estimation for stochastic processes from sampled data, Publ. Inst. Statist. Univ. Paris, 35(3), 51-83.

Prakasa Rao, B. L. S. (1999). Semimartingales and Their Statistical Inference, Monographs on Statistics and Applied Probability, 83, Chapman \& Hall/CRC.

Prakasa Rao, B. L. S. (2000). Statistical Inference for Diffusion Type Processes, ARNOLD.

Shimizu, Y. (2003). M-estimation for discretely observed ergodic diffusion processes with infinitely many jumps, Stat. Infer. Stoc. Proc. (to appear).

Shimizu, Y. (2005). On selection problems of asymptotic filters in inference of stochastic processes with jumps from sampled data (in preparation).

Shimizu, Y. and Yoshida, N. (2002). Estimation for diffusion processes with jumps from discrete observations, Stat. Infer. Stoc. Proc. (to appear). 